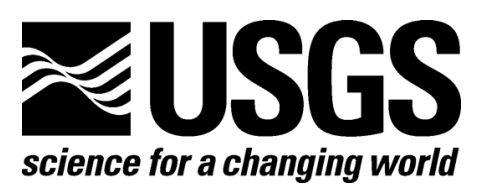

\title{
Global Positioning System Measurements on the Island of Hawai' i: 1997 through 2004
}

By Asta Miklius, Peter Cervelli, Maurice Sako, Michael Lisowski, Susan Owen, Paul Segal, James Foster, Kevan Kamibayashi, and Ben Brooks

Open-File Report 2005-1425

U.S. Department of the Interior

U.S. Geological Survey 


\section{Contents}

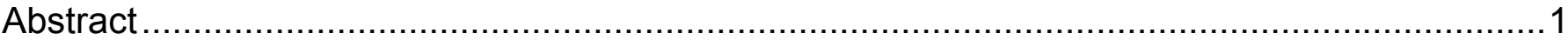

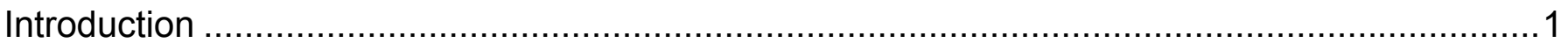

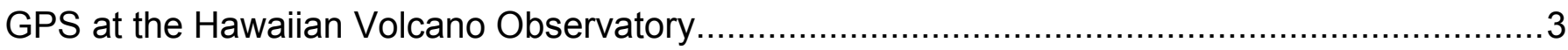

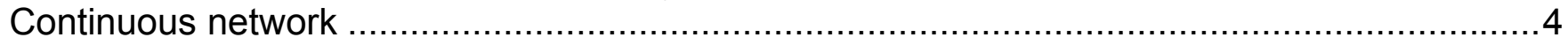

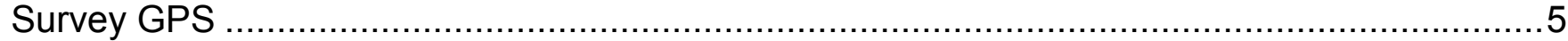

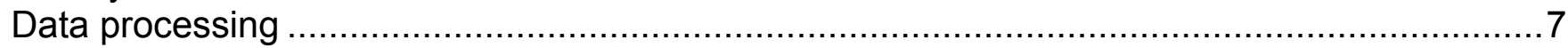

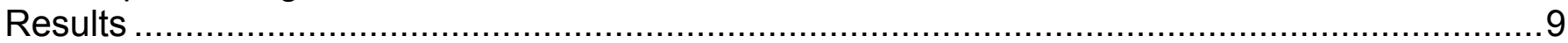

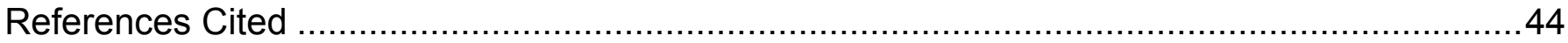

\section{Figures}

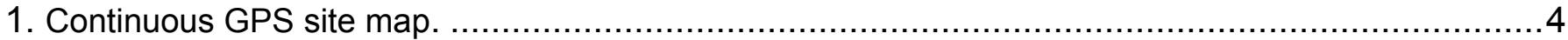

2. Survey GPS site maps $(A)$ Miscellaneous benchmarks and network map locations $(B)$ Kilauea

$(C)$ Mauna Loa $(D)$ Hualalai................................................................ $5-7$

3. Baselines $(A)$ North component of selected Kilauea sites relative to MKPM $(B)$ AHUP-UWEV

(C) MLSP-MOKP.

4. Average horizontal displacement rates $(A)$ 1997-2002 (B) 2002-2004 (C) Hualalai: 1997-2003.

5. Time series of continuous sites $(A)$ AHUP and GOPM $(B)$ HALP and HALR $(C)$ HILO and

$\operatorname{HOLE}(D)$ KAEP and KOSM $(E)$ KTPM and MANE $(F)$ MLPM and NUPM $(G)$ PGF1 and

PGF2 $(H)$ PGF3 and PGF4 (I) PGF5 and PGF6 (J) UWEV and WAOP $(K)$ MKPM $(L)$

AINP and ALEP $(M)$ BLBP and ELEP $(N)$ KFAP and MLCC $(O)$ MLSP and MOKP $(P)$

PAT2 and STEP $(Q)$ TOUO and YEEP.

\section{Tables}

1. Volcanic and tectonic events $(A)$ Geologic events $(B)$ DID's and pauses.............................

2. Continuously recording GPS site codes, locations and notes.

3. Survey GPS benchmark codes, locations and notes.

4. Survey GPS occupation dates $(A)$ Kilauea $(B)$ Mauna Loa $(C)$ Hualalai, Maui and miscellaneous

5. Continuously recording GPS station average velocities $(A)$ 1997-2002 (B) 2002-2004. 


\title{
Global Positioning System Measurements on the Island of Hawai i: 1997 through 2004
}

\author{
By Asta Miklius ${ }^{1}$, Peter Cervelli ${ }^{2}$, Maurice Sako ${ }^{1}$, Michael Lisowski ${ }^{3}$, Susan Owen ${ }^{4}$, Paul \\ Segal $^{5}$, James Foster ${ }^{6}$, Kevan Kamibayashi ${ }^{1}$, and Ben Brooks ${ }^{6}$
}

\section{Abstract}

This report summarizes GPS data and observations collected between 1997 and 2004 on the island of Hawai $i$ with static surveying and continuously recording instruments.

On Kilauea, the long-term deformation field is dominated by steady southeastern velocities of more than $6 \mathrm{~cm} /$ year and uplift of about $2 \mathrm{~cm} / \mathrm{yr}$ at stations on the south flank (with respect to a fixed Pacific Plate). Superimposed on this steady signal are transient displacements associated with magmatic intrusions, earthquakes, and aseismic slip events. The largest of these was the January 30, 1997 dike intrusion and eruption.

GPS instruments near Kilauea's summit also record numerous additional short-term fluctuations associated with variations in magma reservoir pressure and geometry. From mid-1997 through 2001, the dominant signal at the summit was deflationary, with maximum subsidence of $\sim 5$ $\mathrm{cm} / \mathrm{yr}$ south of Kilauea caldera. However, inflation of the magma system was observed from late 2001 to May 2002, and from mid-2003 through 2004. The east rift zone eruption continued at the $\mathrm{Pu}$ 'u 'O`o vent during the entire period of this report.

Mauna Loa Volcano, which most recently erupted in 1984, showed low rates of contraction across the summit caldera and southeasterly motion of the southeast flank from 1997 until May 2002. Reinflation started abruptly in May 2002. Inflation continued through 2004, but at highly variable rates. Extension rates on a baseline across the summit caldera slowed in October 2002 and increased again starting in mid-2003. The most dramatic change during the inflation period, however, was a significant increase in extension rate on longer baselines, from the northwest to southeast flanks, in July 2004.

GPS measurements on Hualalai volcano show no significant motion relative to the Pacific Plate.

\section{Introduction}

Global Positioning System (GPS) measurements on the island of Hawai i began in 1987, and have proven to be an effective tool in monitoring ground deformation associated with volcanic and volcano-tectonic activity in Hawai i (e.g., Dvorak, 1994, Owen et al., 2000a,b, Segall et al.,

\footnotetext{
${ }^{1}$ U.S. Geological Survey, Hawaiian Volcano Observatory, Hawaii National Park, HI

${ }^{2}$ U.S. Geological Survey, Alaska Volcano Observatory, Anchorage, AK

${ }^{3}$ U.S. Geological Survey, Cascades Volcano Observatory, Vancouver, WA

${ }^{4}$ NASA Jet Propulsion Laboratory, Pasadena, CA

${ }^{5}$ Department of Geophysics, Stanford University, Stanford, CA

${ }^{6}$ University of Hawai i, Pacific GPS Facility, Manoa, HI
} 
2001, Cervelli et al., 2002a,b). Sources of deformation on Hawai 1 include the summit and rift zone magma systems of Kilauea and Mauna Loa, and slip along faults beneath their flanks (e.g., Delaney et al., 1998, Owen et al., 2000b, Cervelli and Miklius, 2003).

From 1997 through 2004, Kilauea Volcano erupted almost continuously from the Pu 'u 'O'o vent complex on the east rift zone, continuing the eruption that began in January 1983. There were, however, numerous pauses in eruptive activity (listed in Heliker and Mattox, 2003 and those lasting more than one day in table 1. Most of these lasted only one to two days, but several longer pauses occurred, notably in February 1997 following the Napau crater intrusion and eruption (Owen et al., 2000a) and in September 1999 following a smaller east rift zone dike intrusion (Cervelli et al., 2002b). There have been no discernible pauses since December 2000 (Heliker and Mattox, 2003).

Table 1(A). Geologic events.

\begin{tabular}{|c|c|c|}
\hline Event & Date & Deformation Reference \\
\hline Napau Crater Intrusion and Eruption (Episode 54) & January 30, 1997 & $\begin{array}{l}\text { Owen et al., 2000a; } \\
\text { Desmarais and Segall, in } \\
\text { press }\end{array}$ \\
\hline Lava erupts outside $\mathrm{Pu}$ 'u 'O'o crater & March 28, 1997 & \\
\hline M5.2 Kilauea South Flank Earthquake & June 30, 1997 & Owen et al., 1997 \\
\hline Kilauea Summit Tilt Event/Eruptive surge & January 14, 1998 & Larson et al., 2001 \\
\hline Kilauea South Flank Slip Event & September 19, 1998 & \\
\hline M5.6 Pahala Earthquake & April 16, 1999 & Cervelli et al., 1999 \\
\hline Kilauea Upper East Rift Zone Intrusion & September 12, 1999 & Cervelli et al., $2002 b$ \\
\hline Kilauea Upper East Rift Zone Intrusion & February 23, 2000 & \\
\hline M5.0 Kilauea South Flank Earthquake & April 1, 2000 & \\
\hline Kilauea South Flank Slip Event & November 10, 2000 & Cervelli et al., 2002a \\
\hline $\begin{array}{l}\text { New vent opens at Pu 'u 'O’o, Mother's Day flow, start } \\
\text { of Mauna Loa inflation }\end{array}$ & May 12,2002 & Miklius and Cervelli, 2003 \\
\hline Kilauea South Flank Slip Event & July 3,2003 & \\
\hline M5.0 Kilauea South Flank Earthquake & Aug 26, 2003 & \\
\hline
\end{tabular}

Numerous, short deflation-inflation-deflation (DID) events populate the time series of tilt measurements at the summit and at Pu 'u 'O'o (Cervelli and Miklius, 2003). Table 1(B) lists DID events since the start of concurrent operation of tiltmeters at the summit and at Pu'u ' $\mathrm{O}^{\prime} \mathrm{o}$ in January 2000. These events usually occur on time scales of less than a day, thus they are better imaged in the GPS data with sub-daily solutions. However, several of the larger events can be discerned in daily-average solutions from summit stations, and occasionally coincide with changes in deformation rates at these stations.

There were five earthquakes of magnitude 5 or greater recorded on the island during this reporting period. The largest was a M5.6 earthquake on April 16, 1999 that originated about $9 \mathrm{~km}$ beneath the south flank of Mauna Loa. A M5.1 occurred on July 271998 in the Kohala region. The other three quakes were located between 9 and $10 \mathrm{~km}$ beneath the south flank of Kilauea on June 30, 1997, April 1, 2000, and Aug 26, 2003. 
Table 1(B). Deflation-inflation-deflation (DID) events since 2000 and pauses longer than one day.

\begin{tabular}{ll}
\hline DID's & Pauses \\
\hline September 24 2000 & Jan 31-Feb 24, 1997 \\
April 7 2001 & May 19, 1998 \\
May 20 2001 & July 16, 1998 \\
December 9 2001 & August 12, 1998 \\
April 5 2002 & November 7, 1998 \\
January 21 2003 & May 4, 1999 \\
August 82003 & June 14, 1999 \\
March 4 2004 & Sep 12-23, 1999 \\
March 20 2004 & October 3, 1999 \\
May 15 2004 & November 11, 1999 \\
July 27 2004 & August 23, 2000 \\
& December 15, 2000
\end{tabular}

\section{GPS at the Hawaiian Volcano Observatory}

The Hawaiian Volcano Observatory (HVO) employs several methods of dual-frequency GPS surveying, including static, kinematic, and rapid-static. In collaboration with UNAVCO Inc., HVO also operates a network of single frequency, continuously recording GPS instruments. Several strategies and software packages are used to process the data, including kinematic and hourly average processing. However, we focus here on GPS data and observations collected from 1997 through 2004 on the island of Hawai i with dual-frequency receivers, static surveying and continuously recording instruments, and daily-average solutions. Previous GPS data holdings are summarized in Miklius et al. $(1994,1997)$.

\section{Continuous network}

HVO, Stanford, and the University of Hawaii (UH) began building a network of continuously recording, dual frequency GPS receivers in 1995, with the installation of two sites spanning Kilauea's summit area (UWEV and MANE, figure 1). The number of stations grew steadily, and by the end of 2004, there were more than 30 continuously recording dual-frequency GPS stations monitoring Kilauea and Mauna Loa volcanoes. Table 2 provides the locations and installation dates for the continuous network.

At these sites, GPS antennas are mounted on threaded stainless steel rods set into bedrock (except station UWEV, which is set into the top of a cement vault) and surrounded by an 8-inch diameter PVC pipe filled with cement. 
Data is collected every 30 seconds and transmitted hourly to the observatory via radio modem. We collected data from satellites orbiting higher than 5 degrees above the horizon until 1999, when we decreased the cut-off elevation angle to 0 degrees.

Data from the continuous network is archived both at HVO and at UNAVCO. Data and station equipment information are available from UNAVCO.

The NASA/International GPS Service (IGS) station near the summit of Mauna Kea supplements this network and provides a stable reference point. Although the IGS designation for this site is MKEA, we refer to it as MKPM to avoid confusion with an identically named campaign station. In addition to sites on the Island of Hawai $i$, HVO includes data from GPS stations on other islands in the daily processing. These sites are operated by the UH and the IGS.

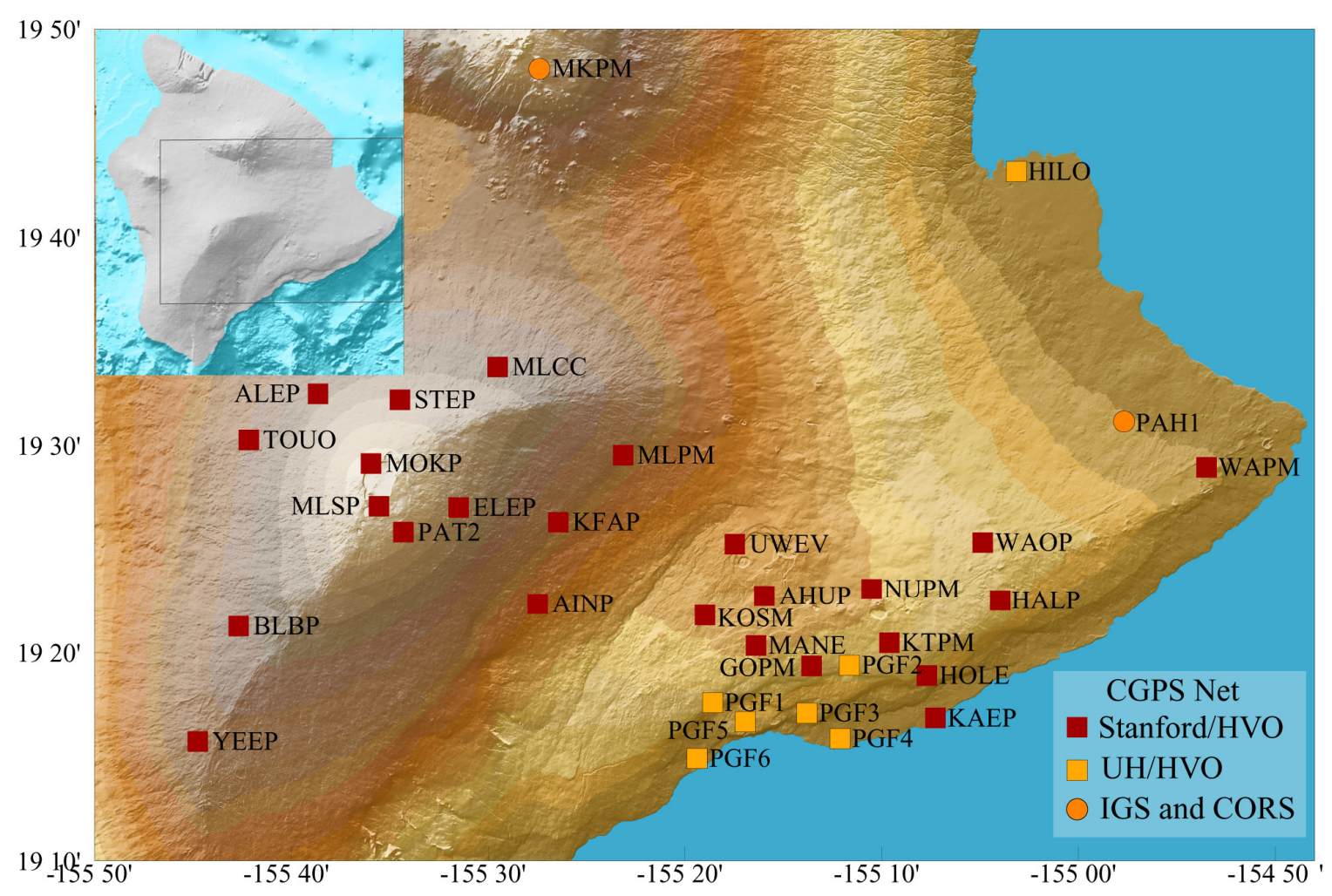

Figure 1. Continuous GPS network on the island of Hawai i. Inset shows area of larger map.

\section{Survey GPS}

During static GPS surveying, the instrument is set up over a benchmark and records data every 30 seconds for anywhere from 8 to 24 hours. At remote, secure locations, such as those accessible only by helicopter, the instruments are usually left on-site to record data over several days. Survey GPS measurements were collected with Trimble 4000SSE, 4000SSI, and 5700 receivers. Data and log sheets are archived at HVO.

Benchmarks occupied with GPS between 1997 and 2004 are listed in table 3 and locations are shown in figure 2 , categorized by networks of benchmarks that are usually occupied during a single campaign. Note that some sites are common to more than one network. Table 4 lists survey dates for each benchmark, in UTC. Sessions often cross a UTC date boundary; the date listed is the 
day in which the bulk of the data is collected. The Kilauea and Mauna Loa networks are occupied at least once yearly. The Hualalai and West Mauna Loa networks are occupied about once every two years. An additional network of densely spaced benchmarks formerly occupied with EDM spans Kilauea's Koa'e fault system. This network, last measured with GPS in May 2003, is not listed here; information on this net is available from the authors.

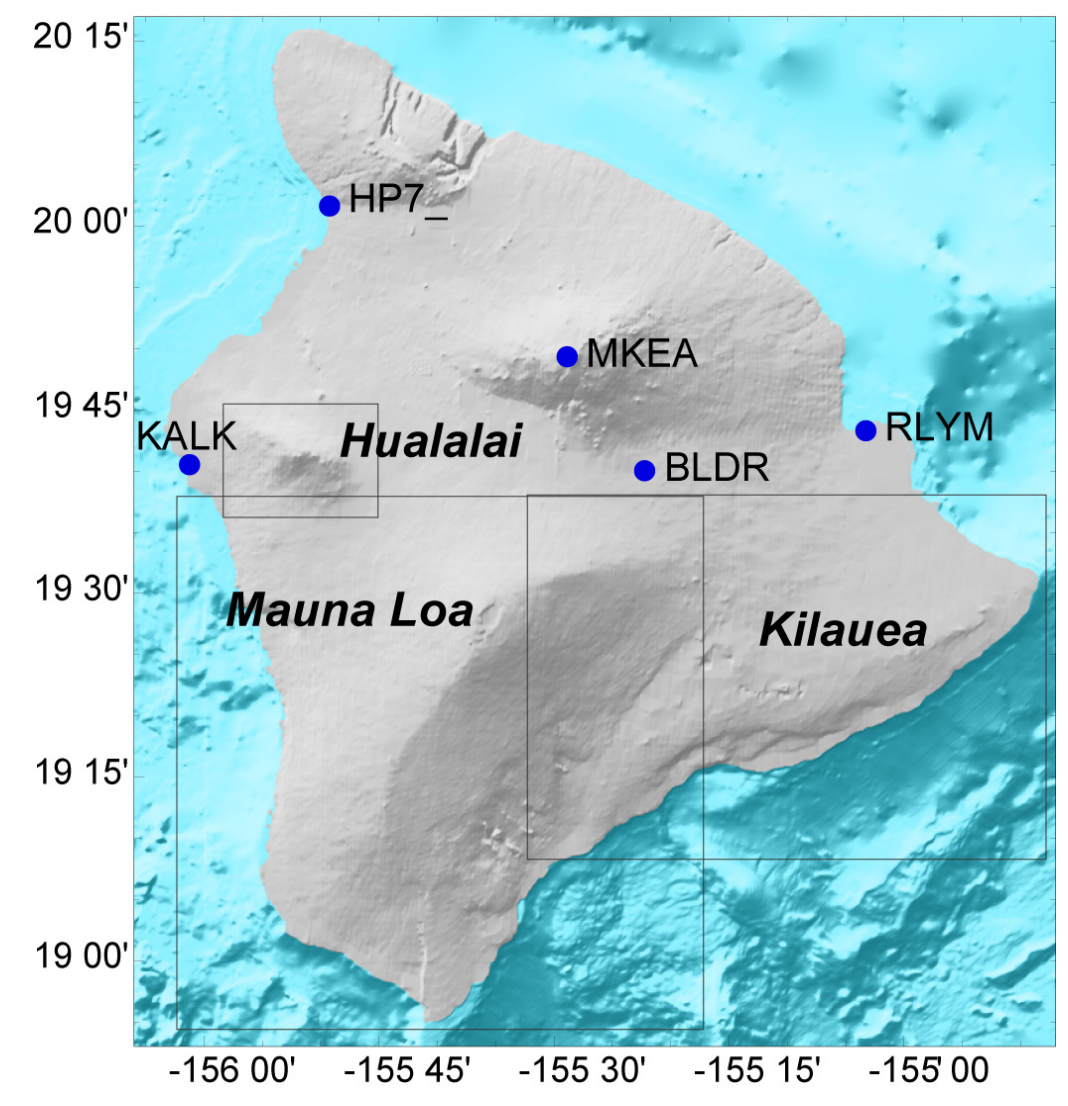

Figure $2(\boldsymbol{A})$. Map of Hawai' $\mathrm{i}$ Island showing the location of "miscellaneous" GPS survey benchmarks (blue dots), which are often occupied with several networks. Boxes indicate the areas of the survey GPS benchmark location maps of figures $2(B)-(D)$. 


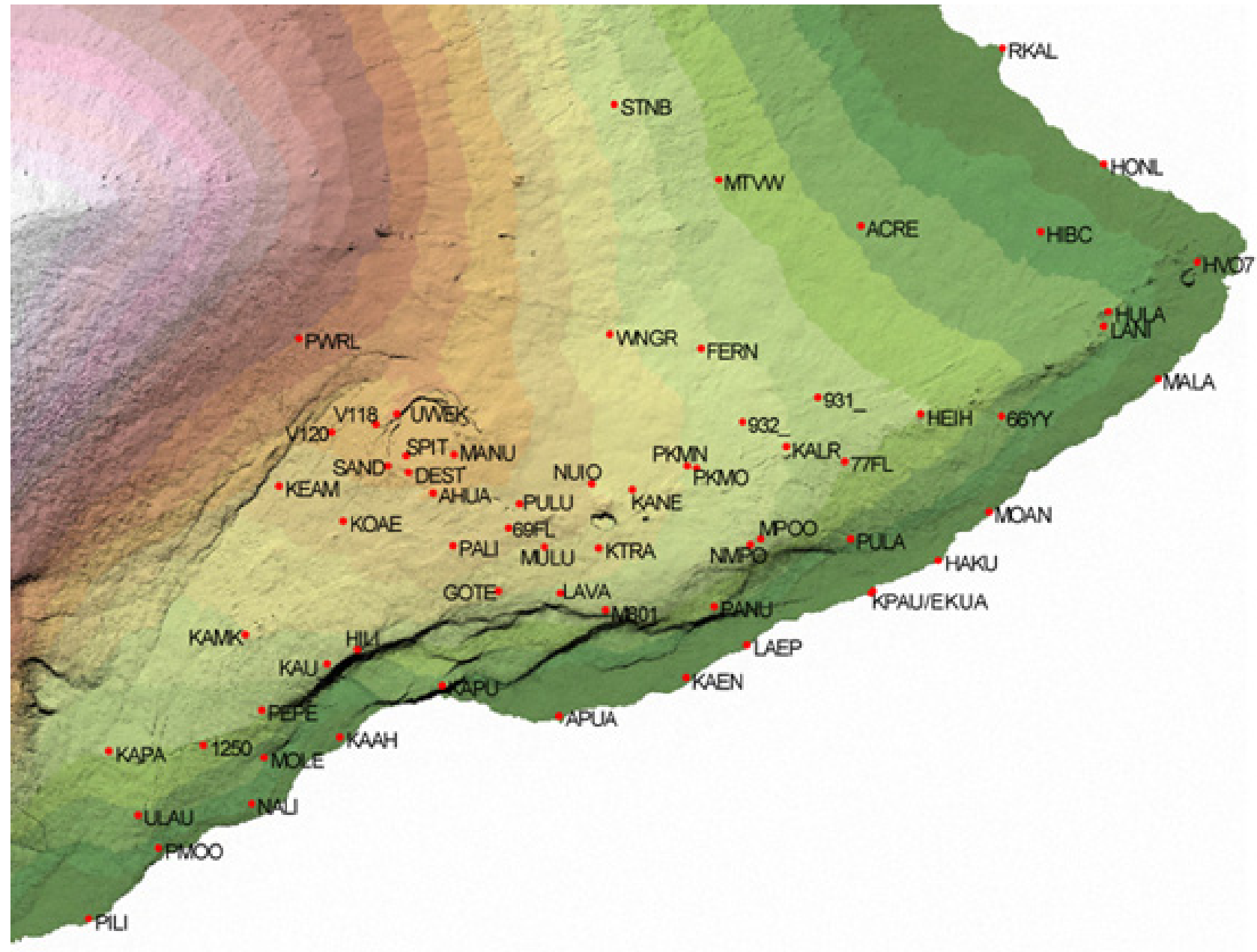

Figure $2(B)$. The Kilauea survey GPS network. 


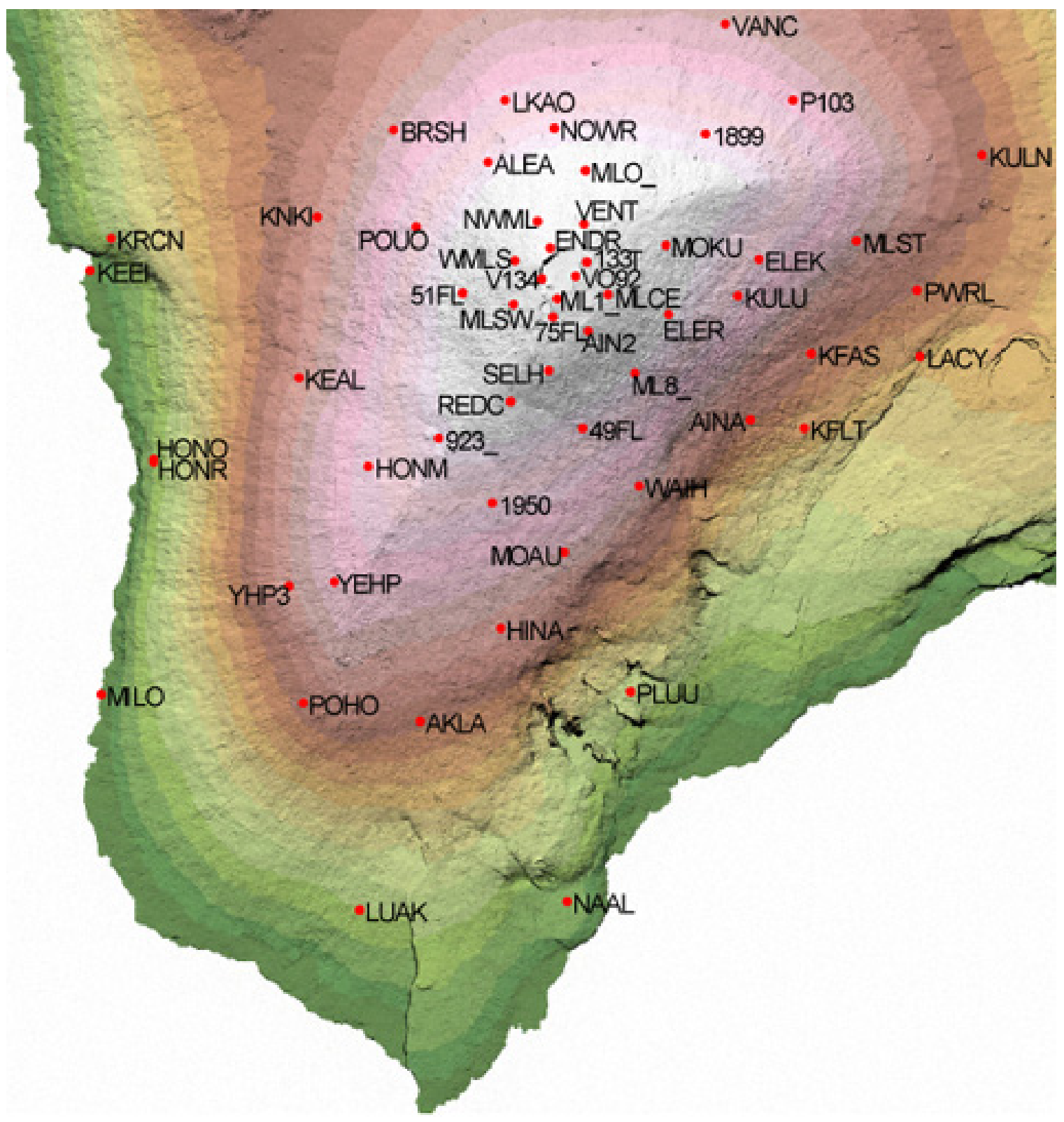

Figure $2(C)$. The Mauna Loa survey GPS network. 


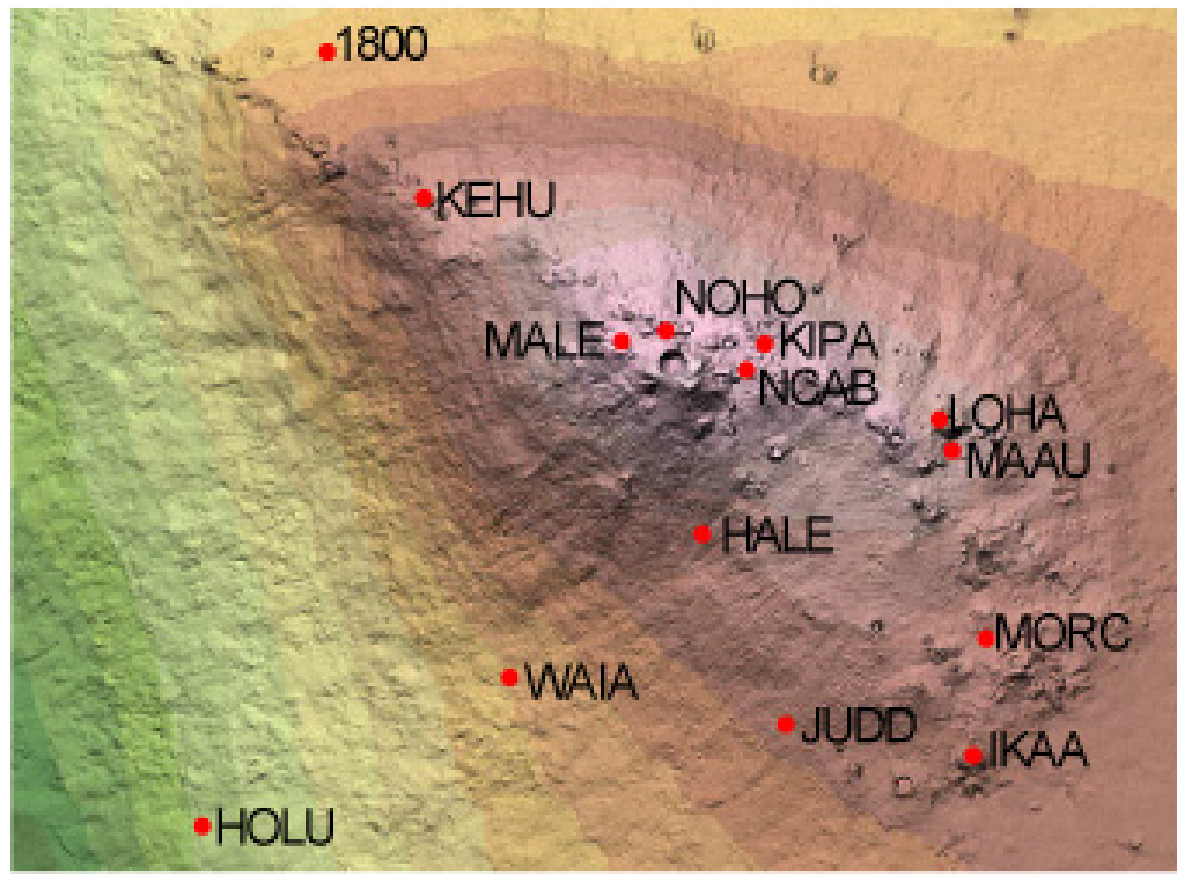

Figure $2(D)$. The Hualalai survey GPS network.

\section{Data Processing}

Data collected by the continuous network are telemetered to HVO in hourly files. Processing the data in near-real-time has been discussed by Larson et al. (2001). The emphasis here, however, will be on daily-average solutions. We process all dual-frequency GPS data using the GIPSY/OASIS II software (Lichten and Border, 1987) with non-fiducial orbits and a precise point positioning strategy (Zumberge et al., 1997). Phase ambiguities are fixed to their integer values when possible. The non-fiducial solutions are then transformed into a global ITRF reference frame with a 7-parameter Helmert transformation using daily parameters provided by the Jet Propulsion Laboratory (JPL). The processing also employs azimuthal tropospheric gradients, which permit more robust estimation of the tropospheric path delays in this region of highly variable, wet tropospheric conditions (Bar-Sever et al., 1998). The estimation of tropospheric gradients requires information from low-angle paths; for this reason an elevation mask of 5 degrees is used. The processing method has evolved over time. However, when a major change of method is implemented, it has been our policy to re-process the older data to create a consistent time series. All of the time series presented here reflect the processing strategy given above. 


\section{Results}

The most significant signal in the Kilauea time series is the large and generally steady seaward motion of stations on the south flank with respect to the Pacific Plate. Superimposed on this steady-state deformation are short-lived events that introduce offsets into the time series (table 1, figures 3(A), 3(B), 5). The largest of these was the January 1997 intrusion and eruption, which caused large perturbations at many sites that lasted for months (Owen et al., 2000a, Desmarais and Segall, in press). Velocities returned to previous rates by mid-1997, and thus the average velocities from 1997 to 2002 shown in figure 4(A) and listed in table 5(A) for Kilauea stations are calculated from July 1997. In late 2001, the continuous GPS network began to record the first sustained inflation of Kilauea since the stations were installed. Inflation ceased with the opening of a new, high-volume vent on Pu'u 'O`o on May 12, 2002 (Miklius and Cervelli, 2003). Inflation resumed in mid-2003 and continued through 2004, with high extension rates across the summit (figure 3(B)) and uplift of summit station UWEV. Baselines that cross the calderas of both Kilauea and Mauna Loa (figure $3(B),(C)$ ) have generally proven to be good proxies for inflation status. These also show numerous short perturbations and changes in extension rates on scales of days to weeks. On Kilauea, most of these are associated with short-lived volcanic events such as pauses in the eruption, or deflation-inflation-deflation cycles that usually end with a surge of lava production at Pu`u `O`o (Cervelli and Miklius, 2003).

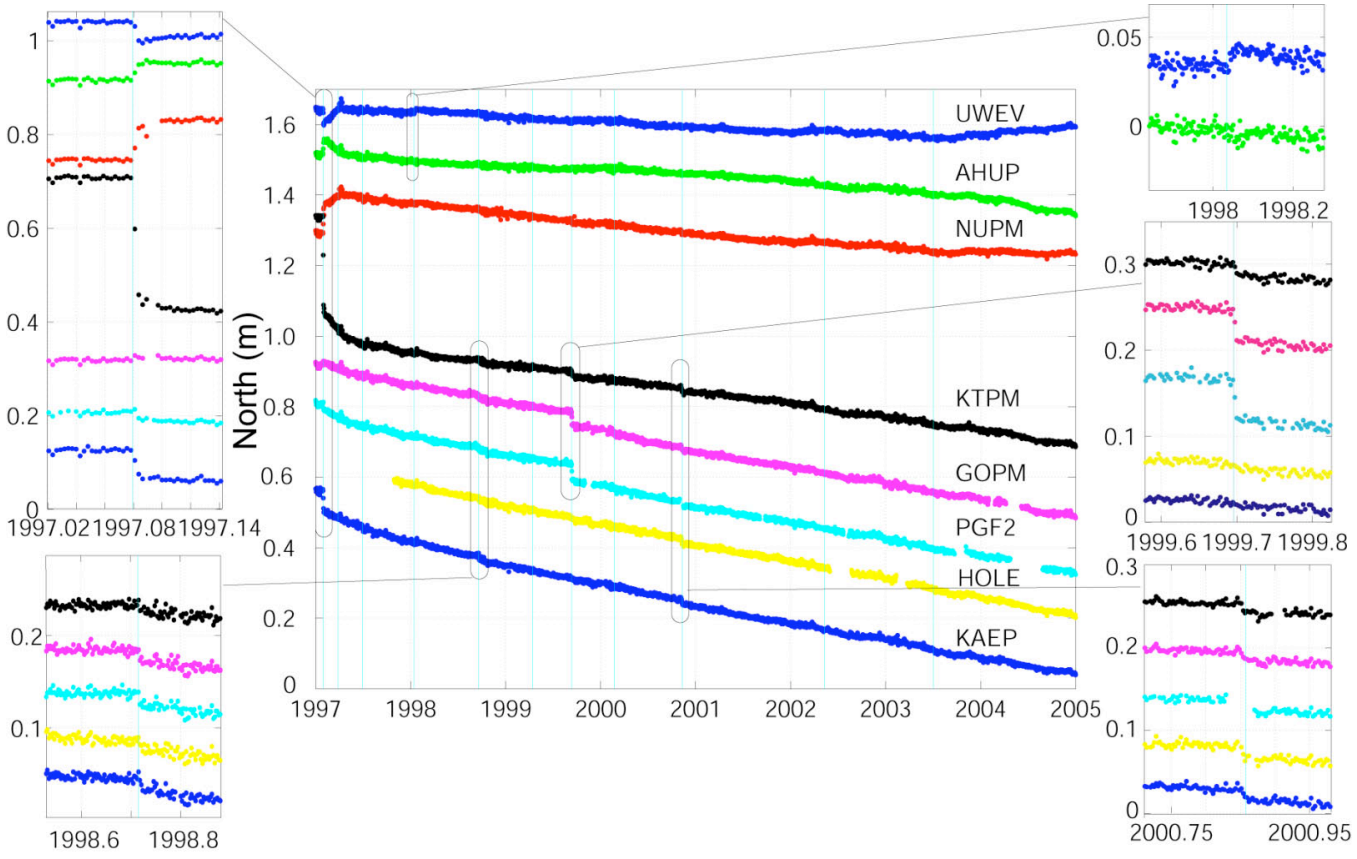

Figure $\mathbf{3}(\mathbf{A})$. The north component of selected continuous GPS stations relative to MKPM. Vertical lines mark geologic events listed in table 1(A). See figure 1 for station locations. 


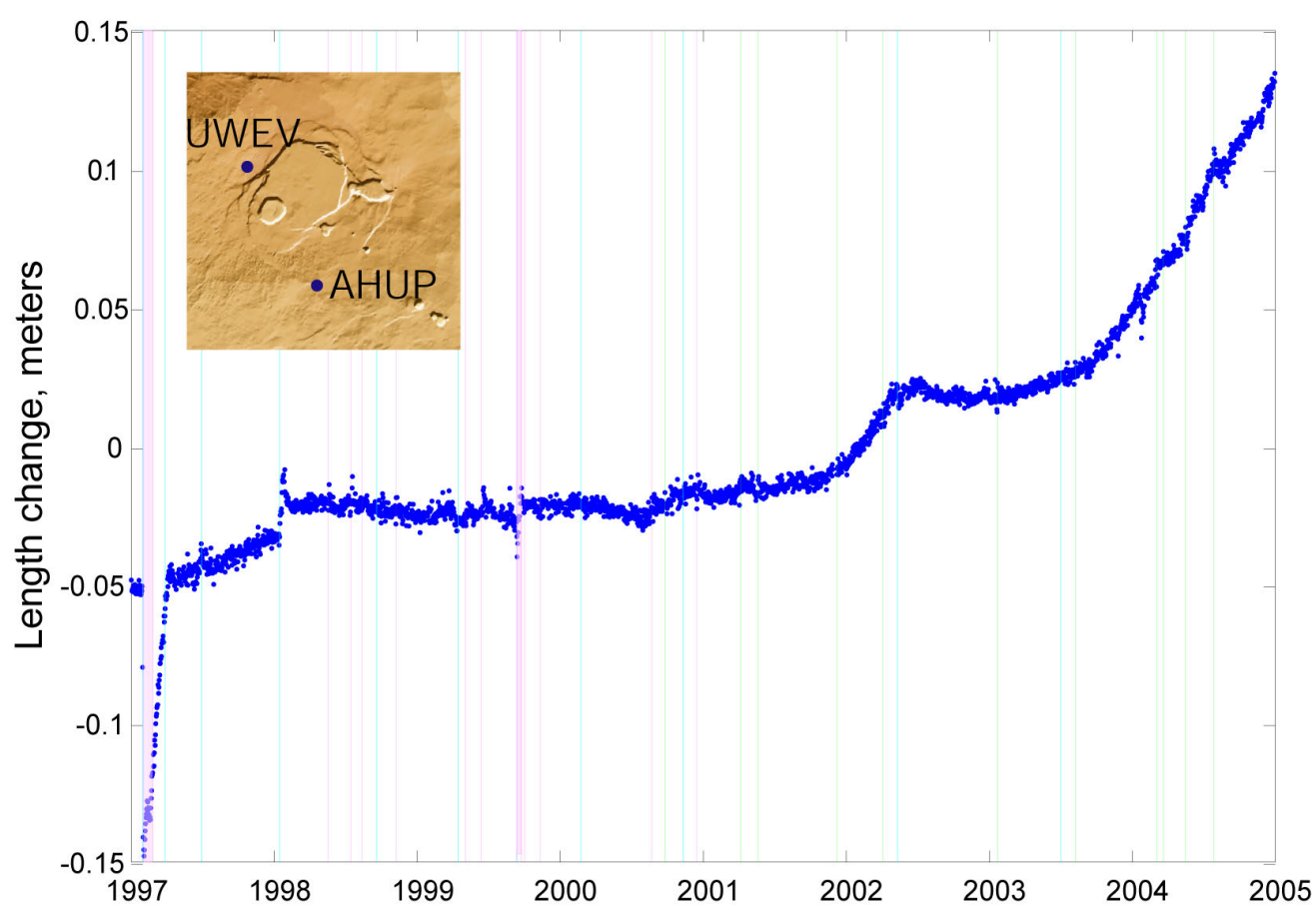

Figure $3(B)$. Line length change between Kilauea summit stations. Light blue vertical lines mark geologic events (table $1(A)$ ), green lines mark DID events (table $1(B)$ ) and pink lines mark pauses (table $1(B)$ ).

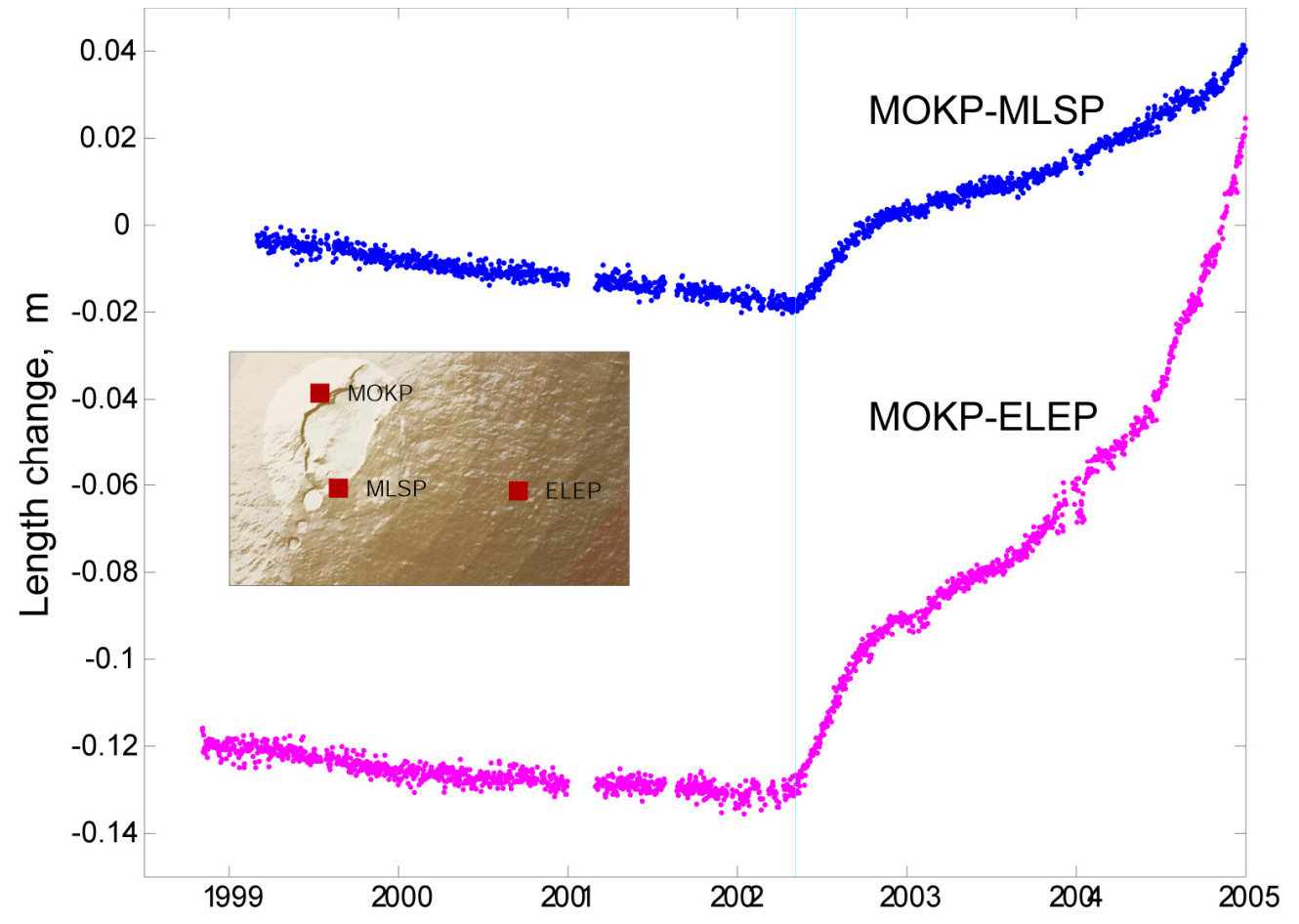

Figure $3(C)$. Line length change between Mauna Loa summit stations, in blue, and flank stations, in pink. The vertical line marks the start of reinflation on May 12, 2002. 
Mauna Loa's summit, which had been extending and uplifting since its most recent eruption in 1984 until mid-1993 (Miklius et al., 1995), showed very low rates of deformation between 1997 and 2002 (figures 3(C), 4(A)). The summit area contracted and subsided, and most stations moved southeastward, with maximum horiontal velocities observed on the upper southeast flank.

Reinflation started abruptly in May 2002 (Miklius and Cervelli, 2003). The initially high inflation rates continued until October 2002, then slowed until mid-2003. Inflation rates accelerated dramatically in mid-2004, best illustrated by the acceleration of extension of longer baselines from the northwest to southeast flanks (fig. 3(C)). This acceleration coincided with the start of a swarm of deep, long-period earthquakes. The high rate of inflation continued through 2004, though the deep seismicity abated at the end of 2003.

GPS measurements on Hualalai volcano show no significant motion relative to the Pacific Plate (fig. 4(C)).

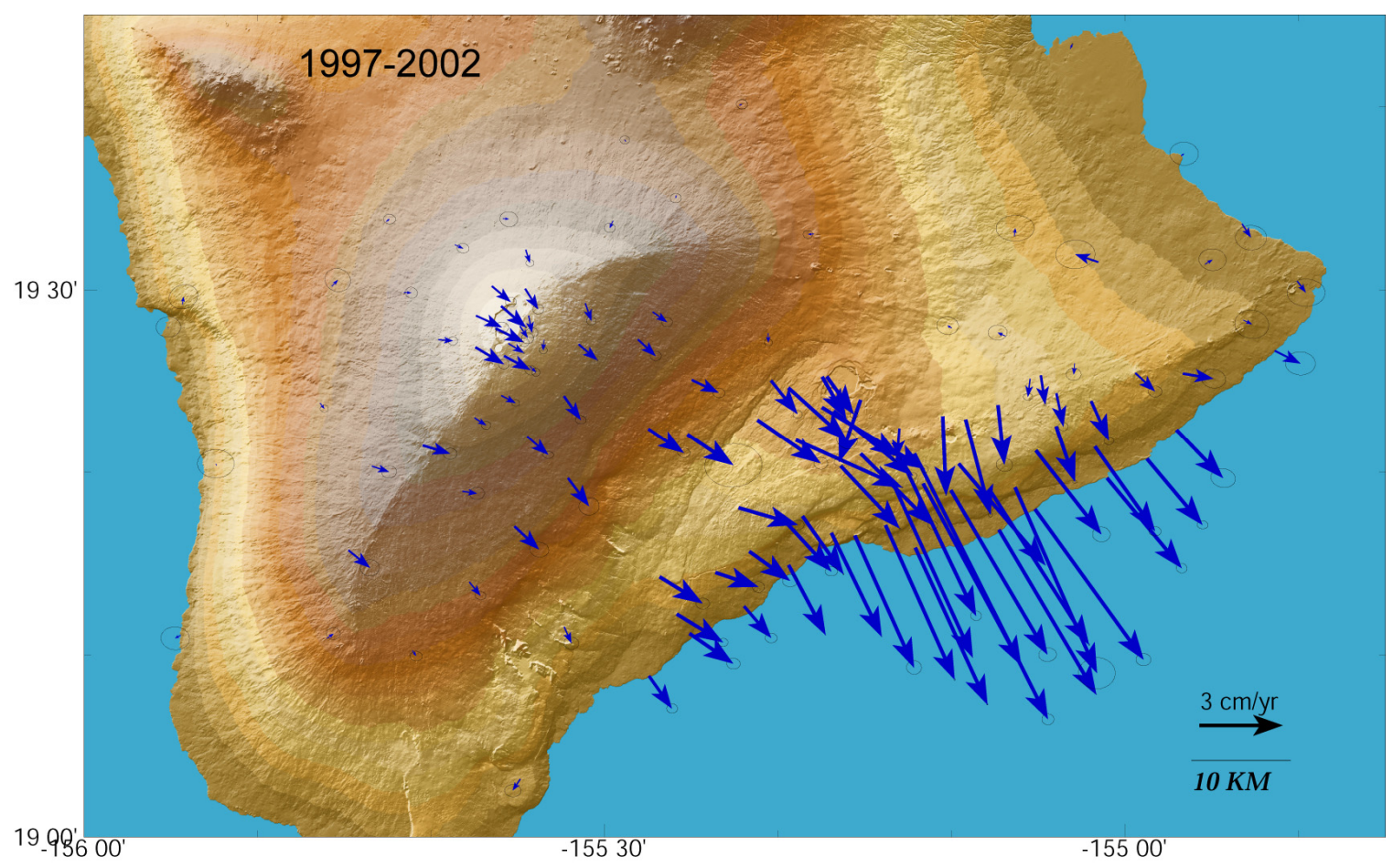

Figure $4(\boldsymbol{A})$. Average horizontal displacement rates from 1997 through 2002. The velocity of the permanent station on the summit on Mauna Kea (MKPM) has been removed (this velocity is listed in table 5). See table 5(A) for specific dates. 2-sigma error ellipses are based on the fit to a linear velocity. 


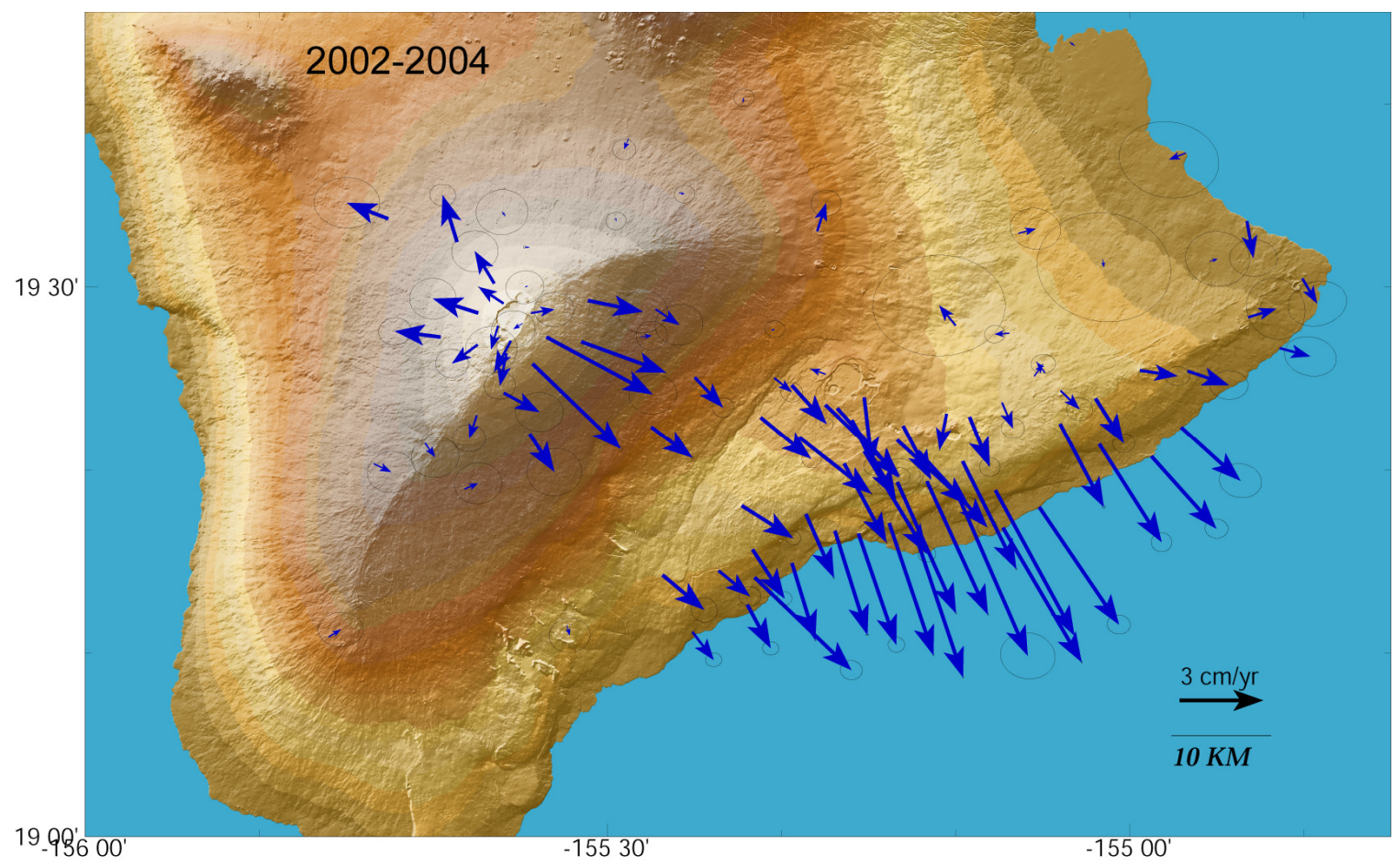

Figure $4(B)$. Average horizontal displacement rates from 2002 through 2004. The velocity of the permanent station on the summit on Mauna Kea (MKPM) has been removed (this velocity is listed in table 5). See table $5(B)$ for specific dates. 2-sigma error ellipses are based on the fit to a linear velocity.

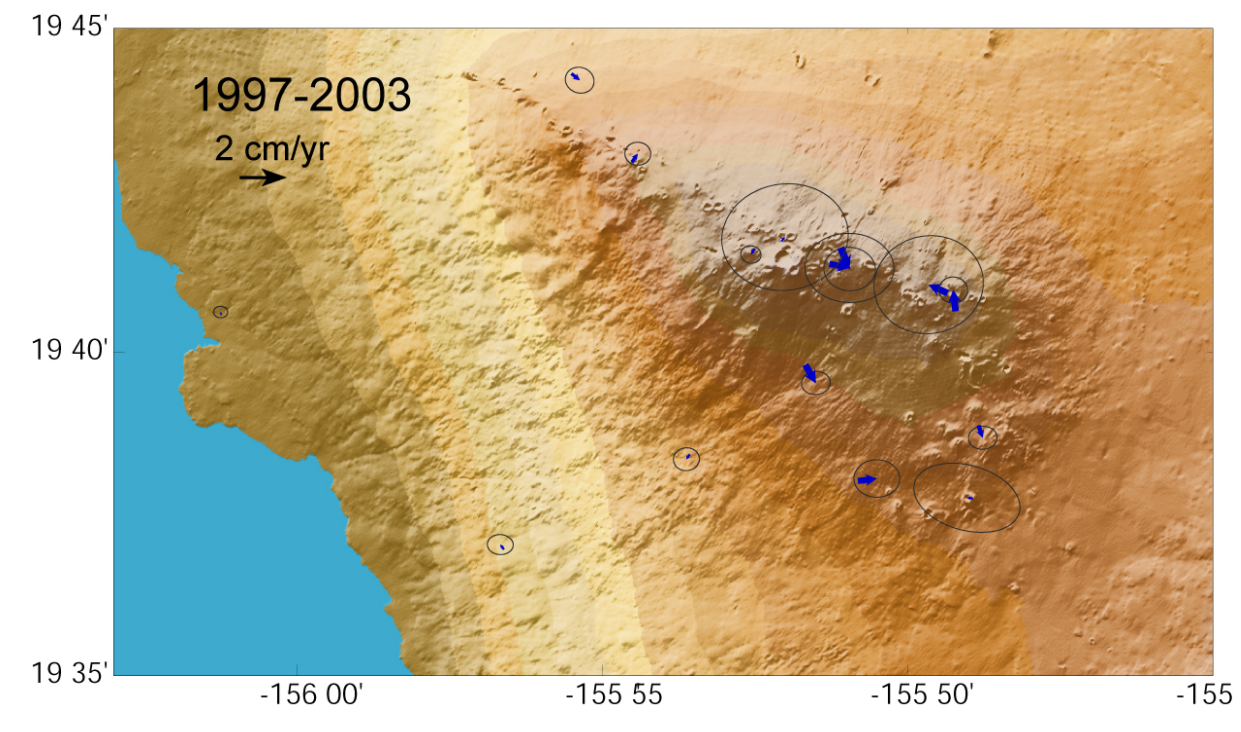

Figure 4 (C). Average horizontal displacement rates on Hualalai Volcano from 1997 to 2003. The velocity of the permanent station on the summit on Mauna Kea (MKPM) has been removed (this velocity is listed in table 5). 2-sigma error ellipses are based on the fit to a linear velocity. 
Time series for each of the continuous sites on Mauna Loa and Kilauea volcanoes are given in figure 5. These time series are plotted relative to the permanent station on the summit on Mauna Kea (MKPM) in order to minimize reference frame error and apparent seasonal and interannual variations that are common to all sites (these can still be seen on the detrended MKPM time series in figure $5(K)$ ). Note that this does introduce a small, spurious vertical signal in all the time series at the 1999-2000 year boundary. The formal errors are not depicted in these time series because their inclusion causes excessive clutter; inspection of the day-to-day scatter gives a qualitative impression of the errors.

The continuous GPS network has proved to be a highly effective tool for monitoring both long-term deformation and the variations in deformation rates that are crucial to understanding the magma system and structure of Hawaii's active volcanoes. 
Table 2. Continuously recording GPS stations: codes, locations in WGS84, and notes

\begin{tabular}{|l|c|c|c|c|}
\hline code & longitude & latitude & installation date & \\
\hline AHUP & -155.2661 & 19.3791 & $7 / 20 / 1996$ & \\
\hline AINP & -155.4580 & 19.3727 & $10 / 8 / 2002$ & \\
\hline ALEP & -155.6441 & 19.5412 & $7 / 15 / 2004$ & \\
\hline BLBP & -155.7114 & 19.3546 & $6 / 12 / 2004$ & \\
\hline ELEP & -155.5249 & 19.4503 & $10 / 22 / 1998$ & \\
\hline GOPM & -155.2224 & 19.3223 & $8 / 15 / 1996$ & \\
\hline HALP & -155.0665 & 19.3756 & $12 / 28 / 1999$ & Overrun by lava flows in May 2002, replaced by HALR \\
\hline HALR & -155.0665 & 19.3756 & $2 / 1 / 2003$ & \\
\hline HILO & -155.0527 & 19.7192 & $6 / 15 / 1997$ & \\
\hline HOLE & -155.1285 & 19.3149 & $10 / 30 / 1997$ & \\
\hline KAEP & -155.1214 & 19.2809 & $7 / 20 / 1996$ & \\
\hline KFAP & -155.4406 & 19.4384 & $7 / 18 / 2004$ & \\
\hline KOSM & -155.3165 & 19.3633 & $5 / 15 / 1997$ & \\
\hline KTPM & -155.1601 & 19.3413 & $11 / 1 / 1996$ & \\
\hline MANE & -155.2733 & 19.3391 & $7 / 29 / 1995$ & \\
\hline MKPM & -155.4563 & 19.8014 & $9 / 26 / 1996$ & NASA IGS station \\
\hline MLCC & -155.4920 & 19.5627 & $7 / 2 / 2004$ & \\
\hline MLPM & -155.3859 & 19.4920 & $1 / 28 / 1997$ & \\
\hline MLSP & -155.5924 & 19.4512 & $3 / 2 / 1999$ & \\
\hline MOKP & -155.5994 & 19.4853 & $11 / 4 / 1998$ & \\
\hline NUPM & -155.1753 & 19.3847 & $10 / 10 / 1996$ & \\
\hline PAT2 & -155.5717 & 19.4304 & $11 / 1 / 2002$ & \\
\hline PGF1 & -155.3101 & 19.2933 & $9 / 6 / 1996$ & \\
\hline PGF2 & -155.1941 & 19.3232 & $9 / 6 / 1996$ & \\
\hline PGF3 & -155.2302 & 19.2850 & $11 / 3 / 1996$ & \\
\hline PGF4 & -155.2018 & 19.2646 & $9 / 7 / 1996$ & \\
\hline PGF5 & -155.2823 & 19.2779 & $9 / 1 / 1996$ & \\
\hline PGF6 & -155.3233 & 19.2486 & $9 / 6 / 1996$ & \\
\hline STEP & -155.5748 & 19.5366 & $10 / 11 / 2002$ & \\
\hline TOUO & -155.7028 & 19.5043 & $7 / 10 / 2004$ & \\
\hline UWEV & -155.2911 & 19.4209 & $5 / 27 / 1995$ & \\
\hline WAOP & -155.0811 & 19.4221 & $12 / 18 / 1999$ & \\
\hline WAPM & -154.8916 & 19.4823 & $5 / 12 / 2004$ & \\
\hline YEEP & -155.7462 & 19.2622 & $8 / 18 / 2004$ & \\
\hline
\end{tabular}


Table 3. Survey GPS benchmarks: codes, locations in WGS84, and notes

\begin{tabular}{|c|c|c|c|}
\hline Code & Latitude & Longitude & Notes \\
\hline 1250 & -155.3937 & 19.2419 & \\
\hline 133T & -155.5731 & 19.4765 & \\
\hline 1800 & -155.9250 & 19.7383 & \\
\hline 1899 & -155.4920 & 19.5627 & reset to continuous station MLCC 2004 \\
\hline 1950 & -155.6366 & 19.3161 & \\
\hline 49FL & -155.5745 & 19.3663 & \\
\hline$\overline{51 \mathrm{FL}}$ & -155.6597 & 19.4545 & \\
\hline $66 \mathrm{YY}$ & -154.9446 & 19.4239 & \\
\hline 69FL & -155.2227 & 19.3608 & \\
\hline $75 \mathrm{FL}$ & -155.5968 & 19.4398 & \\
\hline 77FL & -155.0328 & 19.3987 & \\
\hline 923 & -155.6748 & 19.3579 & \\
\hline 931 & -155.0487 & 19.4330 & reset to continuous station WAOP 1999 \\
\hline 932 & -155.0913 & 19.4191 & \\
\hline$\overline{\mathrm{ACRE}}$ & -155.0257 & 19.5252 & \\
\hline $\mathrm{AHUA}$ & -155.2661 & 19.3791 & reset to continuous station AHUP 1996 \\
\hline AIN2 & -155.5717 & 19.4304 & reset to continuous station PAT2 2004 \\
\hline AINA & -155.4580 & 19.3733 & reset to continuous station AINP 2002 \\
\hline AKLA & -155.6848 & 19.1705 & \\
\hline ALEA & -155.6438 & 19.5411 & reset to continuous station ALEP 2004 \\
\hline APUA & -155.1927 & 19.2599 & reset to continuous station PGF4 1996 \\
\hline BLDR & -155.3705 & 19.6680 & \\
\hline BRSH & -155.7096 & 19.5620 & \\
\hline DEST & -155.2800 & 19.3897 & \\
\hline EKUA & -155.0160 & 19.3294 & reset from KPAU \\
\hline ELEK & -155.4540 & 19.4800 & \\
\hline ELER & -155.5163 & 19.4428 & reset to continuous station ELEP 1998 \\
\hline ENDR & -155.5989 & 19.4856 & reset from HVO93, reset to continuous station MOKP 1998 \\
\hline FERN & -155.1153 & 19.4581 & \\
\hline GOTE & -155.2281 & 19.3265 & reset to continuous station GOPM 1996 \\
\hline HAIL & -156.3433 & 20.8512 & \\
\hline HAKU & -154.9791 & 19.3459 & \\
\hline HALE & -155.8612 & 19.6634 & \\
\hline HANA & -156.0145 & 20.7959 & \\
\hline $\mathrm{HEIH}$ & -154.9902 & 19.4242 & \\
\hline $\mathrm{HIBC}$ & -154.9233 & 19.5235 & \\
\hline $\mathrm{HILI}$ & -155.3073 & 19.2945 & reset to continuous station PGF1 1996 \\
\hline HINA & -155.6299 & 19.2332 & \\
\hline $\mathrm{HKHI}$ & -156.1794 & 20.7350 & \\
\hline HOLU & -155.9435 & 19.6159 & \\
\hline HONL & -154.8877 & 19.5599 & BM may be unstable \\
\hline HONM & -155.7236 & 19.3390 & \\
\hline HONO & -155.8730 & 19.3416 & reset to HONR 1999 \\
\hline HONR & -155.8727 & 19.3397 & reset from HONO \\
\hline HP7 & -155.8207 & 20.0268 & \\
\hline HULA & -154.8844 & 19.4806 & \\
\hline HVO7 & -154.8346 & 19.5078 & \\
\hline IKAA & -155.8155 & 19.6290 & \\
\hline JUDD & -155.8468 & 19.6335 & \\
\hline $\mathrm{KAAH}$ & -155.3168 & 19.2471 & reset to continuous station PGF6 1996 \\
\hline KAEN & -155.1215 & 19.2812 & reset to continuous station KAEP 1996 \\
\hline KALK & -156.0206 & 19.6762 & \\
\hline KALR & -155.0661 & 19.4059 & \\
\hline
\end{tabular}




\begin{tabular}{|c|c|c|c|}
\hline Code & Latitude & Longitude & Notes \\
\hline KAMK & -155.3713 & 19.3010 & \\
\hline KANE & -155.1534 & 19.3818 & \\
\hline KAPA & -155.4472 & 19.2378 & \\
\hline KAPU & -155.2596 & 19.2757 & \\
\hline KAU_ & -155.3244 & 19.2862 & \\
\hline KEAL & -155.7732 & 19.3969 & \\
\hline KEAM & -155.3532 & 19.3814 & \\
\hline KEEI & -155.9193 & 19.4654 & \\
\hline KEHU & -155.9085 & 19.7156 & \\
\hline KFAS & -155.4163 & 19.4180 & reset to continuous station KFAP 2004 \\
\hline KFLT & -155.4206 & 19.3682 & \\
\hline KHUA & -156.2965 & 20.6787 & \\
\hline KIPA & -155.8515 & 19.6935 & \\
\hline KNAE & -156.1439 & 20.8623 & \\
\hline KNKI & -155.7623 & 19.5031 & \\
\hline KOAE & -155.3165 & 19.3633 & reset to continuous station KOSM 1997 \\
\hline KOLE & -156.2562 & 20.7073 & \\
\hline KOLI & -155.5411 & 19.6344 & \\
\hline KPAU & -155.0175 & 19.3281 & overrun by lava 2001 \\
\hline KRCN & -155.9056 & 19.4868 & \\
\hline KTRA & -155.1717 & 19.3505 & reset to continuous site KTPM 1997 \\
\hline KULN & -155.2992 & 19.5508 & \\
\hline KULU & -155.4680 & 19.4550 & \\
\hline LACY & -155.3403 & 19.4174 & \\
\hline LAEP & -155.0868 & 19.2996 & \\
\hline LANI & -154.8868 & 19.4725 & \\
\hline LAVA & -155.1929 & 19.3261 & reset to continuous station PGF2 1996 \\
\hline LEIE & -156.0473 & 20.6531 & \\
\hline LKAO & -155.6322 & 19.5831 & \\
\hline LOHA & -155.8224 & 19.6819 & \\
\hline LOOK & -156.2340 & 20.7368 & \\
\hline LUAK & -155.7249 & 19.0450 & \\
\hline LUAL & -156.3060 & 20.6096 & \\
\hline M801 & -155.1674 & 19.3170 & \\
\hline MAAU & -155.8202 & 19.6772 & \\
\hline MALA & -154.8563 & 19.4447 & \\
\hline MALE & -155.8752 & 19.6933 & \\
\hline MANU & -155.2541 & 19.3998 & \\
\hline MILO & -155.9071 & 19.1845 & \\
\hline MKEA & -155.4810 & 19.8228 & \\
\hline ML1_ & -155.5935 & 19.4518 & reset to continuous station MLSP 1998 \\
\hline ML8 & -155.5393 & 19.4033 & \\
\hline MLCE & -155.5582 & 19.4547 & \\
\hline $\mathrm{MLO}$ & -155.5757 & 19.5365 & reset to continuous station STEP 2002 \\
\hline MLST & -155.3857 & 19.4924 & reset to continuous station MLPM 1997 \\
\hline MLSW & -155.6243 & 19.4479 & \\
\hline MOAN & -154.9508 & 19.3724 & \\
\hline MOAU & -155.5866 & 19.2841 & \\
\hline MOKU & -155.5187 & 19.4878 & \\
\hline MOLE & -155.3593 & 19.2358 & \\
\hline MORC & -155.8139 & 19.6478 & \\
\hline MPOO & -155.0799 & 19.3564 & overrun by lava 1997 \\
\hline MTVW & -155.1065 & 19.5490 & \\
\hline
\end{tabular}




\begin{tabular}{|c|c|c|c|}
\hline \multicolumn{4}{|c|}{ Table 3 continued. Survey GPS benchmarks } \\
\hline Code & Latitude & Longitude & Notes \\
\hline MULU & -155.2025 & 19.3504 & \\
\hline NAAL & -155.5810 & 19.0530 & \\
\hline NALI & -155.3662 & 19.2109 & \\
\hline NCAB & -155.8546 & 19.6894 & \\
\hline NIAN & -156.2449 & 20.7724 & \\
\hline NMPO & -155.0859 & 19.3534 & replacement for MPOO, overrun by lava 2002 \\
\hline $\mathrm{NOHO}$ & -155.8677 & 19.6952 & \\
\hline NOWR & -155.5979 & 19.5646 & \\
\hline $\mathrm{NUIO}$ & -155.1762 & 19.3851 & \\
\hline$\overline{N W M L}$ & -155.6082 & 19.5033 & \\
\hline $\mathrm{P} 103$ & -155.4315 & 19.5857 & \\
\hline PALI & -155.2542 & 19.3505 & \\
\hline PANU & -155.1059 & 19.3196 & reset to continuous site HOLE 1997 \\
\hline PEPE & -155.3612 & 19.2611 & \\
\hline $\mathrm{PILI}$ & -155.4574 & 19.1475 & \\
\hline PKMN & -155.1222 & 19.3952 & replacement for PKMO \\
\hline PKMO & -155.1172 & 19.3938 & overrun by lava 1998 \\
\hline PLUU & -155.5390 & 19.1922 & \\
\hline PMOO & -155.4185 & 19.1862 & \\
\hline $\mathrm{POHO}$ & -155.7667 & 19.1817 & \\
\hline POLI & -156.3015 & 20.7124 & \\
\hline POUO & -155.6929 & 19.4976 & \\
\hline PULA & -155.0293 & 19.3570 & \\
\hline PULU & -155.2170 & 19.3736 & \\
\hline PUUI & -156.3873 & 20.6827 & \\
\hline PWRL & -155.3430 & 19.4608 & \\
\hline REDC & -155.6251 & 19.3836 & \\
\hline RKAL & -154.9464 & 19.6218 & \\
\hline RLYM & -155.0548 & 19.7228 & reset to continuous station HILO 1997 \\
\hline SAND & -155.2914 & 19.3929 & \\
\hline SELH & -155.5991 & 19.4038 & \\
\hline SPIT & -155.2812 & 19.3985 & \\
\hline STNB & -155.1658 & 19.5889 & overgrown with large trees \\
\hline ULAU & -155.4307 & 19.2038 & \\
\hline UWEK & -155.2868 & 19.4211 & displaced autumn 2001, reset as UWER \\
\hline UWER & -155.2868 & 19.4211 & reset of UWEK, possibly not well tied to ground \\
\hline V118 & -155.2988 & 19.4152 & \\
\hline V120 & -155.3236 & 19.4109 & \\
\hline V134 & -155.6046 & 19.4648 & \\
\hline VANC & -155.4796 & 19.6349 & \\
\hline VENT & -155.5763 & 19.5010 & \\
\hline V092 & -155.5815 & 19.4669 & \\
\hline WAIA & -155.8927 & 19.6403 & \\
\hline WAIH & -155.5352 & 19.3281 & \\
\hline WMLS & -155.6236 & 19.4764 & \\
\hline$\overline{\text { WNGR }}$ & -155.1667 & 19.4651 & \\
\hline YEHP & -155.7461 & 19.2621 & reset to continuous station YEEP 2004 \\
\hline YHP3 & -155.7776 & 19.2588 & \\
\hline
\end{tabular}




\begin{tabular}{|c|c|c|c|c|c|c|c|c|c|c|c|}
\hline Code & 1997 & & & 1998 & & 1999 & 2000 & 2001 & 2002 & 2003 & 2004 \\
\hline '1250' & & $6 / 22-23 / 1997$ & & & $6 / 27-29 / 1998$ & $6 / 26-28 / 1999$ & $6 / 24-26 / 2000$ & $6 / 30-7 / 1 / 2001$ & $7 / 8 / 2002$ & $9 / 13-15 / 2003$ & $8 / 13-15 / 2004$ \\
\hline '66YY' & & $\begin{array}{r}\text { 6/15/1997, } \\
7 / 16 / 1997\end{array}$ & & & $6 / 23 / 1998$ & $6 / 22,29 / 1999$ & $6 / 21-22 / 2000$ & $6 / 27 / 2001$ & $7 / 16 / 2002$ & $\begin{array}{r}\text { 9/17/2003, } \\
10 / 17 / 2003\end{array}$ & $9 / 22-23 / 2004$ \\
\hline '69FL' & $\begin{array}{r}1 / 16- \\
17 / 1997, \\
2 / 4 / 1997\end{array}$ & 6/18-19/1997 & 9/9/1997 & $\begin{array}{r}\text { 1/9/1998, } \\
4 / 16 / 1998\end{array}$ & 6/26/1998 & $\begin{array}{r}6 / 26-27 / 1999 \\
9 / 14 / 1999 \\
\end{array}$ & $\begin{array}{r}3 / 1-2 / 2000 \\
6 / 24-25 / 2000\end{array}$ & $7 / 7-8 / / 2001$ & $\begin{array}{r}7 / 10- \\
11 / 2002 \\
\end{array}$ & $8 / 21 / 2003$ & $\begin{array}{r}4 / 28 \\
30 / 2004 \\
9 / 21-23 / 2004 \\
\end{array}$ \\
\hline '77FL' & $2 / 5 / 1997$ & $\begin{array}{r}6 / 17- \\
18 / 1997,7 / 3- \\
7 / 1997 \\
\end{array}$ & & & $6 / 23-24 / 1998$ & $6 / 22-23 / 1999$ & $6 / 20-21 / 2000$ & $6 / 26-27 / 2001$ & $7 / 4-5 / 2002$ & 9/9-102003 & $8 / 11-12 / 2004$ \\
\hline '931_' & $2 / 6 / 1997$ & $6 / 17-18 / 1997$ & & & $6 / 23-24 / 1998$ & $6 / 22 / 1999$ & $6 / 20-21 / 2000$ & $6 / 26-27 / 2001$ & & & \\
\hline '932_' & $\begin{array}{r}\text { 2/6/1997, } \\
5 / 8-13 / 1997\end{array}$ & $\begin{array}{r}6 / 17- \\
18 / 1997,7 / 3- \\
7 / 1997 \\
\end{array}$ & & & 6/23-24/1998 & 6/22-23/1999 & $6 / 20-21 / 2000$ & $6 / 26-27 / 2001$ & $7 / 4-5 / 2002$ & $9 / 12 / 2003$ & $8 / 11-12 / 2004$ \\
\hline 'ACRE' & & 6/17-18/1997 & & & $6 / 29 / 1998$ & $6 / 22,30 / 1999$ & $6 / 22,26 / 2000$ & $6 / 26 / 2001$ & $7 / 17 / 2002$ & 9/19/2003 & $12 / 8 / 2004$ \\
\hline 'AHUA' & & & 9/18/1997 & & & & & & & & \\
\hline 'APUA' & & $6 / 19-20 / 1997$ & & & & & & & & & \\
\hline 'DEST' & $\begin{array}{r}\text { 1/21/1997, } \\
\text { 2/4/1997 }\end{array}$ & $6 / 21-23 / 1997$ & 9/10/1997 & $\begin{array}{r}1 / 10-12 \\
1 / 16,4 / 17- \\
18 / 1998 \\
\end{array}$ & 6/27-28/1998 & 6/26-28/1999 & $6 / 24-26 / 2000$ & $6 / 30-7 / 2 / 2001$ & $\begin{array}{r}7 / 10- \\
11 / 2002 \\
\end{array}$ & $8 / 21 / 2003$ & $4 / 23 / 2004$ \\
\hline 'EKUA' & & & & & & & & $3 / 8 / 2001$ & $7 / 4-5 / 2002$ & 9/9-10/2003 & $8 / 11-12 / 2004$ \\
\hline 'FERN' & 2/20/1997 & $\begin{array}{l}\text { 6/18/1997, } \\
6 / 24 / 1997\end{array}$ & 8/14/1997 & & 6/23-24/1998 & $6 / 22-23 / 1999$ & $6 / 21-22 / 2000$ & $7 / 18 / 2001$ & $\begin{array}{r}7 / 25- \\
28 / 2002\end{array}$ & $\begin{array}{r}10 / 31- \\
11 / 1 / 2003\end{array}$ & $9 / 15-16 / 2004$ \\
\hline 'GOTE' & $\begin{array}{r}1 / 18- \\
23 / 1997 \\
\end{array}$ & & & & & & & & & & \\
\hline 'HAKU' & & $\begin{array}{r}6 / 17- \\
18 / 1997,7 / 1- \\
3 / 1997 \\
\end{array}$ & & & 6/23-24/1998 & 6/22-23/1999 & $6 / 20-21 / 2000$ & $6 / 26-27 / 2001$ & $7 / 4-5 / 2002$ & 9/9-10/2003 & $8 / 11-12 / 2004$ \\
\hline 'HEIH' & & $6 / 17-18 / 1997$ & & & 6/23-24/1998 & $6 / 22-23 / 1999$ & $6 / 20-21 / 2000$ & $6 / 26-27 / 2001$ & $7 / 4-5 / 2002$ & 9/9-10/2003 & $8 / 11-12 / 2004$ \\
\hline 'HIBC' & & 6/17/1997 & & & $\begin{array}{r}6 / 22 / 1998 \\
8 / 7 / 1998 \\
\end{array}$ & $6 / 23,28 / 1999$ & $6 / 20,22 / 2000$ & $\begin{array}{r}6 / 27 / 2001 \\
7 / 3 / 2001 \\
\end{array}$ & $7 / 16 / 2002$ & $9 / 12 / 2003$ & $7 / 26,29 / 2004$ \\
\hline 'HILI' & $1 / 22 / 1997$ & $6 / 21-22 / 1997$ & & & & & & & & & \\
\hline 'HONL' & & $\begin{array}{l}\text { 6/17/1997, } \\
6 / 25 / 1997\end{array}$ & & & $\begin{array}{r}\text { 6/30/1998, } \\
8 / 7 / 1998\end{array}$ & $6 / 23,29 / 1999$ & $6 / 28-29 / 2000$ & & $7 / 16 / 2002$ & $10 / 15 / 2003$ & $\begin{array}{r}8 / 23,27 / 2004 \\
9 / 15 / 2004\end{array}$ \\
\hline 'HULA' & & & & & & & & & $\begin{array}{r}7 / 17- \\
18 / 2002 \\
\end{array}$ & $9 / 20 / 2003$ & $9 / 22-23 / 2004$ \\
\hline 'HVO7' & & 6/17/1997 & & & $\begin{array}{r}\text { 6/23/1998, } \\
8 / 9 / 1998\end{array}$ & $6 / 21,30 / 1999$ & $6 / 28-29 / 2000$ & $7 / 5 / 2001$ & $7 / 16 / 2002$ & $\begin{array}{r}\text { 9/16/2003, } \\
\text { 10/15/2003 }\end{array}$ & $\begin{array}{l}6 / 28 / 2004 \\
8 / 26 / 2004\end{array}$ \\
\hline
\end{tabular}


Table 4a continued. Kilauea GPS surveys

\begin{tabular}{|c|c|c|c|c|c|c|c|c|c|c|c|}
\hline Code & 1997 & & & 1998 & & 1999 & 2000 & 2001 & 2002 & 2003 & 2004 \\
\hline 'KAAH' & & $6 / 21-23 / 1997$ & & & & & & & & & \\
\hline 'KAEN' & $\begin{array}{r}1 / 16- \\
17 / 1997 \\
\end{array}$ & & 9/16/1997 & & & & & $8 / 9-12 / 2001$ & & & \\
\hline 'KALR' & 2/5/1997 & $\begin{array}{r}6 / 17- \\
18 / 1997,7 / 4- \\
7 / 1997 \\
\end{array}$ & & & 6/23-24/1998 & $6 / 22-23 / 1999$ & $6 / 20-21 / 2000$ & $6 / 26-27 / 2001$ & $7 / 4-5 / 2002$ & $9 / 12 / 2003$ & $8 / 11-12 / 2004$ \\
\hline 'KAMK' & & 6/21-23/1997 & & & 6/27-29/1998 & 6/24-25/1999 & $6 / 24-26 / 2000$ & $6 / 30-7 / 2 / / 2001$ & $7 / 6-8 / 2002$ & $9 / 13-14 / 2003$ & 8/13-15/2004 \\
\hline 'KANE' & $\begin{array}{r}2 / 7,3 / 22- \\
29,4 / 2-24, \\
5 / 7-30 / 1997 \\
\end{array}$ & $6 / 19-20 / 1997$ & $\begin{array}{r}8 / 2- \\
11 / 21 / 1997 \\
\end{array}$ & & $6 / 25-26 / 1998$ & $\begin{array}{r}6 / 24-25 / 1999 \\
10 / 14-31 / 1999 \\
\end{array}$ & $6 / 22-23 / 2000$ & $6 / 28 / 2001$ & $7 / 4-5 / 2002$ & $9 / 12 / 2003$ & $8 / 11-12 / 2004$ \\
\hline 'KAPA' & & $6 / 21-22 / 1997$ & & & $6 / 27-28 / 1998$ & $\begin{array}{r}5 / 1-3 / 1999 \\
6 / 26-28 / 1999 \\
\end{array}$ & $6 / 19-20 / 2000$ & $6 / 5-6 / 2001$ & $\begin{array}{r}7 / 10- \\
11 / 2002 \\
\end{array}$ & & $6 / 16-17 / 2004$ \\
\hline 'KAPU' & & 6/19-20/1997 & & & 6/25-26/1998 & 6/24-25/1999 & $6 / 22-23 / 2000$ & $6 / 28-29 / 2001$ & $7 / 6-8 / 2002$ & $9 / 10 / 2003$ & $8 / 13-14 / 2004$ \\
\hline 'KAU_' & & 6/19-20/1997 & & & $6 / 25-26 / 1998$ & 6/24-25/1999 & $6 / 22-23 / 2000$ & $6 / 28-29 / 2001$ & $7 / 6-8 / 2002$ & & \\
\hline 'KEAM' & 2/21/1997 & $6 / 21-22 / 1997$ & & & $6 / 27-28 / 1998$ & $6 / 26-28 / 1999$ & $6 / 27-29 / 2000$ & $6 / 5-6 / 2001$ & $\begin{array}{r}7 / 10- \\
11 / 2002 \\
\end{array}$ & $\begin{array}{r}4 / 3 / 2003,8 / 23- \\
26 / 2003 \\
\end{array}$ & $6 / 15-16 / 2004$ \\
\hline 'KOAE' & $\begin{array}{r}1 / 19- \\
20 / 1997 \\
3 / 5- \\
4 / 23 / 1997\end{array}$ & $6 / 27-30 / 1997$ & & & & & & & & & \\
\hline 'KPAU' & 2/5/1997 & $\begin{array}{r}6 / 17- \\
18 / 1997,7 / 1- \\
3 / 1997 \\
\end{array}$ & & & $6 / 23-24 / 1998$ & 6/22-23/1999 & & $\begin{array}{r}3 / 8 / 2001 \\
6 / 26-27 / 2001 \\
\end{array}$ & & & \\
\hline KTRA & 2/7/1997 & & & & & & & & & & \\
\hline 'LAEP' & 2/19/1997 & $\begin{array}{r}\text { 6/20/1997, } \\
\text { 7/1/1997 }\end{array}$ & $\begin{array}{l}\text { 10/1/1997, } \\
\text { 11/11/1997 }\end{array}$ & & $6 / 26 / 1998$ & $6 / 25,30 / 1999$ & $6 / 24-26 / 2000$ & $6 / 27 / 2001$ & $7 / 12 / 2002$ & & $9 / 21-23 / 2004$ \\
\hline 'LANI' & & $\begin{array}{l}\text { 6/17/1997, } \\
6 / 23 / 1997\end{array}$ & & & 6/30/1998 & 6/28/1999 & $6 / 28-29 / 2000$ & $6 / 28 / 2001$ & $7 / 16 / 2002$ & $9 / 17 / 2003$ & $8 / 20 / 2004$ \\
\hline 'LAVA' & $1 / 16 / 1997$ & $6 / 20 / 1997$ & 9/16/1997 & & & & & & & & \\
\hline 'M801' & 2/19/1997 & $\begin{array}{r}\text { 6/19/1997, } \\
\text { 7/1/1997 } \\
\end{array}$ & 10/1/1997 & & 6/24-30/1998 & $6 / 25 / 1999$ & $6 / 23,26 / 2000$ & $8 / 5-8,12 / 2001$ & $7 / 12 / 2002$ & $7 / 5,8-10 / 2003$ & \\
\hline 'MALA' & & $\begin{array}{c}6 / 15 / 1997 \\
6 / 19 / 1997\end{array}$ & & & $6 / 22,25 / 1998$ & $6 / 21,24 / 1999$ & $6 / 19,28 / 2000$ & $6 / 28 / 2001$ & $7 / 16 / 2002$ & $\begin{array}{r}9 / 10 / 2003 \\
10 / 7 / 2003\end{array}$ & $7 / 15,28 / 2004$ \\
\hline
\end{tabular}


Table 4a continued. Kilauea GPS surveys

\begin{tabular}{|c|c|c|c|c|c|c|c|c|c|c|c|}
\hline Code & 1997 & & & 1998 & & 1999 & 2000 & 2001 & 2002 & 2003 & 2004 \\
\hline 'MANU' & $\begin{array}{r}\text { 1/17/1997, } \\
2 / 4 / 1997 \\
\end{array}$ & 6/24-25/1997 & 9/10/1997 & $\begin{array}{r}\text { 1/8,1/21, } \\
4 / 16 / 1998 \\
\end{array}$ & 6/28-29/1998 & $6 / 25 / 1999$ & $\begin{array}{r}3 / 1-2 / 2000 \\
6 / 24-25 / 2000\end{array}$ & $7 / 3-5 / 2001$ & $\begin{array}{r}3 / 19- \\
20 / 2002 \\
7 / 10- \\
11 / 2002\end{array}$ & $8 / 21 / 2003$ & $4 / 28-30 / 2004$ \\
\hline 'MOAN' & & $\begin{array}{r}\text { 6/15,25/1997, } \\
7 / 16 / 1997 \\
\end{array}$ & & & $6 / 22 / 1998$ & 6/28-29/1999 & $6 / 19 / 2000$ & $\begin{array}{r}6 / 25 / 2001 \\
7 / 3 / 2001 \\
\end{array}$ & $7 / 16 / 2002$ & $9 / 17 / 2003$ & $\begin{array}{r}7 / 22 / 2004, \\
8 / 24-25 / 2004 \\
\end{array}$ \\
\hline 'MOLE' & & & & & & & & $6 / 30-7 / 2 / 2001$ & $7 / 6-8 / 2002$ & & $8 / 13-14 / 2004$ \\
\hline 'MPOO' & $\begin{array}{r}2 / 6,3 / 22 \\
5 / 8-9,5 / 11- \\
13 / 1997 \\
\end{array}$ & $\begin{array}{r}6 / 19- \\
20 / 1997,7 / 1- \\
3 / 1997 \\
\end{array}$ & & & & & & & & & \\
\hline 'MTVW' & & $\begin{array}{r}\text { 6/19/1997 } \\
6 / 24 / 1997 \\
\end{array}$ & & & $6 / 22,25 / 1998$ & $6 / 23,29 / 1999$ & $6 / 27-28 / 2000$ & $6 / 25 / 2001$ & $7 / 17 / 2002$ & $10 / 25 / 2003$ & $7 / 20,27 / 2004$ \\
\hline 'MULU' & $\begin{array}{r}2 / 7, \\
3 / 22 / 1997 \\
\end{array}$ & 6/19-20/1997 & & & & $\begin{array}{r}6 / 24-25 / 1999 \\
9 / 14 / 1999 \\
\end{array}$ & $\begin{array}{r}3 / 4-6 / 2000 \\
6 / 22-23 / 2000 \\
\end{array}$ & $6 / 28-29 / 2001$ & $7 / 4-5 / 2002$ & $9 / 12 / 2003$ & $8 / 13-15 / 2004$ \\
\hline 'NALI' & & $6 / 21-23 / 1997$ & & & $6 / 27-29 / 1998$ & 6/26-28/1999 & $6 / 24-26 / 2000$ & $6 / 30-7 / 1 / 2001$ & $7 / 6-8 / 2002$ & $9 / 13-14 / 2003$ & $8 / 13-15 / 2004$ \\
\hline 'NMPO' & & & & & $7 / 22-23 / 1998$ & 6/24-25/1999 & $6 / 20-21 / 2000$ & $6 / 28-29 / 2001$ & & & \\
\hline 'NUIO' & 2/7/1997 & & & & & & & & & & \\
\hline 'PALI' & $\begin{array}{r}1 / 22- \\
23 / 1997, \\
2 / 8-10 / 1997 \\
\end{array}$ & $6 / 21-22 / 1997$ & 9/9/1997 & $\begin{array}{r}\text { 1/13/1998, } \\
4 / 18 / 1998 \\
\end{array}$ & 6/27-28/1998 & 6/26-28/1999 & 6/23-26/2000 & $6 / 28-29 / 2001$ & $\begin{array}{r}7 / 10- \\
11 / 2002 \\
\end{array}$ & $\begin{array}{r}5 / 17-19 / 2003 \\
8 / 21 / 2003 \\
\end{array}$ & $9 / 21-23 / 2004$ \\
\hline 'PANU' & $\begin{array}{r}\text { 2/1-4/1997, } \\
3 / 22 / 1997\end{array}$ & $\begin{array}{r}6 / 19- \\
20 / 1997,7 / 4- \\
7 / 1997 \\
\end{array}$ & & & 6/25-26/1998 & & & & & & \\
\hline 'PEPE' & & $6 / 21-23 / 1997$ & & & $6 / 25-26 / 1998$ & 6/24-25/1999 & $6 / 22-23 / 2000$ & $6 / 28-29 / 2001$ & $7 / 6-8 / 2002$ & $9 / 12-13 / 2003$ & $8 / 13-15 / 2004$ \\
\hline 'PILI' & & 6/19-20/1997 & & & 6/27-28/1998 & 5/4/1999 & $6 / 24-26 / 2000$ & $6 / 30-7 / 1 / 2001$ & & $9 / 13-15 / 2003$ & $6 / 24-25 / 2004$ \\
\hline 'PKMN' & & & & & $6 / 26 / 1998$ & $6 / 24-25 / 1999$ & $6 / 22-23 / 2000$ & $6 / 28-29 / 2001$ & $7 / 4 / 2002$ & $9 / 12 / 2003$ & $8 / 11-12 / 2004$ \\
\hline 'PKMO' & $\begin{array}{r}1 / 30 \\
2 / 6,5 / 7-9, \\
5 / 11- \\
30 / 1997 \\
\end{array}$ & & & & & & & & & & \\
\hline 'PMOO' & & $6 / 21-23 / 1997$ & & & $6 / 27-29 / 1998$ & 6/26-28/1999 & $6 / 24-26 / 2000$ & $6 / 30-7 / 1 / 2001$ & $7 / 6-8 / 2002$ & $9 / 13-14 / 2003$ & $8 / 13-15 / 2004$ \\
\hline
\end{tabular}


Table 4a continued. Kilauea GPS surveys

\begin{tabular}{|c|c|c|c|c|c|c|c|c|c|c|c|}
\hline Code & 1997 & & & 1998 & & 1999 & 2000 & 2001 & 2002 & 2003 & 2004 \\
\hline 'PULA' & 2/5/1997 & $\begin{array}{r}6 / 17- \\
18 / 1997,7 / 1- \\
3 / 1997 \\
\end{array}$ & & & $6 / 23-24 / 1998$ & $6 / 22-23 / 1999$ & $6 / 20-21 / 2000$ & $6 / 26-27 / 2001$ & $7 / 4-5 / 2002$ & $9 / 9-10 / 2003$ & $8 / 11-12 / 2004$ \\
\hline 'PULU' & $\begin{array}{r}1 / 22-23 \\
1 / 31- \\
2 / 3,2 / 28- \\
3 / 21 / 1997 \\
\end{array}$ & $\begin{array}{r}\text { 6/24- } \\
\text { 25/1997, } \\
7 / 1 / 1997 \\
\end{array}$ & 9/6-8/1997 & $\begin{array}{r}\text { 1/8,1/21, } \\
4 / 16 / 1998 \\
\end{array}$ & 6/28-29/1998 & $\begin{array}{r}\text { 6/26-28/1999, } \\
9 / 21-22 / 1999 \\
\end{array}$ & $\begin{array}{r}4 / 30-5 / 1 / 2000 \\
6 / 22-23 / 2000 \\
\end{array}$ & $7 / 3-5 / 2001$ & $\begin{array}{r}3 / 20- \\
21 / 2002 \\
7 / 10- \\
11 / 2002 \\
\end{array}$ & $8 / 21 / 2003$ & $\begin{array}{r}4 / 28- \\
30 / 2004, \\
9 / 21-23 / 2004 \\
\end{array}$ \\
\hline 'PWRL' & $\begin{array}{r}1 / 18-21 \\
2 / 27,3 / 1- \\
5 / 1997 \\
\end{array}$ & $6 / 11-12 / 1997$ & $\begin{array}{r}9 / 20- \\
22 / 1997 \\
\end{array}$ & $1 / 13 / 1998$ & $6 / 6-8 / 1998$ & 6/8-10/1999 & $5 / 25-26 / 2000$ & $\begin{array}{l}\text { 5/31/2001, } \\
6 / 2-4 / 2001 \\
\end{array}$ & $7 / 25 / 2002$ & $3 / 11-12 / 2003$ & $9 / 28-29 / 2004$ \\
\hline 'RKAL' & & $\begin{array}{l}\text { 6/15/1997, } \\
\text { 6/24/1997 }\end{array}$ & & & $6 / 24,29 / 1998$ & $6 / 22 / 1999$ & $6 / 28-30 / 2000$ & $6 / 28 / 2001$ & $7 / 16 / 2002$ & 9/19/2003 & \\
\hline 'SAND' & $\begin{array}{r}1 / 19-20 \\
2 / 1-3,3 / 6- \\
21 / 1997\end{array}$ & $6 / 21-22 / 1997$ & $\begin{array}{r}9 / 6- \\
10 / 1997\end{array}$ & $\begin{array}{r}1 / 10- \\
12 / 1998 \\
1 / 16- \\
19 / 1998 \\
4 / 17- \\
18 / 1998\end{array}$ & $6 / 27-28 / 1998$ & $6 / 26-28 / 1999$ & $6 / 24-25 / 2000$ & $6 / 30-7 / 2 / 2001$ & $\begin{array}{r}3 / 19- \\
20 / 2002 \\
7 / 10- \\
11 / 2002\end{array}$ & $8 / 21 / 2003$ & $4 / 24-26 / 2004$ \\
\hline 'SPIT' & & & & & & & & & $\begin{array}{r}12 / 3- \\
6 / 2002 \\
\end{array}$ & $1 / 28-31 / 2003$ & \\
\hline 'STNB' & & $6 / 26 / 1997$ & & & $\begin{array}{r}6 / 30- \\
7 / 1 / 1998 \\
\end{array}$ & & & & & & \\
\hline 'ULAU' & & $6 / 21-23 / 1997$ & & & $6 / 27-29 / 1998$ & $6 / 26-28 / 1999$ & $6 / 24-26 / 2000$ & $6 / 30-7 / 2 / 2001$ & $7 / 6-8 / 2002$ & & \\
\hline 'UWEK' & $\begin{array}{r}1 / 22 / 1997 \\
2 / 10- \\
12 / 1997\end{array}$ & 6/24-25/1997 & 9/12/1997 & $\begin{array}{r}\text { 1/9/1998, } \\
1 / 23 / 1998\end{array}$ & 6/27-28/1998 & $6 / 26-28 / 1999$ & $6 / 27-29 / 2000$ & $7 / 3-5 / 2001$ & & & \\
\hline 'UWER' & & & & & & & & & $\begin{array}{r}5 / 23- \\
24 / 2002 \\
12 / 3- \\
6 / 2002\end{array}$ & $1 / 28-31 / 2003$ & $\begin{array}{r}4 / 20 / 2004, \\
7 / 16 / 2004\end{array}$ \\
\hline 'V118' & & & & & & & & & & & $4 / 29 / 2004$ \\
\hline 'V120' & & 6/18-19/1997 & & & $6 / 25 / 1998$ & $6 / 24 / 1999$ & $6 / 22-23 / 2000$ & $6 / 5-6 / 2001$ & $\begin{array}{r}3 / 20- \\
21 / 2002 \\
7 / 10- \\
11 / 2002 \\
\end{array}$ & $8 / 23-25 / 2003$ & $\begin{array}{r}6 / 15- \\
16 / 2004, \\
9 / 28-29 / 2004 \\
\end{array}$ \\
\hline 'WNGR' & 2/19/1997 & $6 / 23-24 / 1997$ & $8 / 14 / 1997$ & & $6 / 24, / 26 / 1998$ & 7/1-2/1999 & $6 / 21-22 / 2000$ & $6 / 29 / 2001$ & & $9 / 24 / 2003$ & $\begin{array}{r}7 / 21 / 2004, \\
8 / 31 / 2004\end{array}$ \\
\hline
\end{tabular}




\begin{tabular}{|c|c|c|c|c|c|c|c|c|}
\hline Code & 1997 & 1998 & 1999 & 2000 & 2001 & 2002 & 2003 & 2004 \\
\hline '133T' & $6 / 5 / 1997$ & $5 / 28-29 / 1998$ & $5 / 26 / 1999$ & $5 / 9 / 2000$ & $6 / 19 / 2001$ & $4 / 4 / 2002$ & $3 / 18 / 2003$ & $6 / 2-3 / 2004$ \\
\hline '1899' & $6 / 12 / 1997$ & 6/4-5/1998 & $5 / 25 / 1999$ & $5 / 24-25 / 2000$ & $\begin{array}{c}5 / 30- \\
6 / 1 / 2001\end{array}$ & $7 / 2-24 / 2002$ & $3 / 12-13 / 2003$ & $7 / 1-2 / 2004$ \\
\hline '1950' & & $5 / 31 / 1998$ & $5 / 28 / 1999$ & $5 / 12 / 2000$ & $6 / 22 / 2001$ & $4 / 4-5 / 2002$ & $3 / 20 / 2003$ & $6 / 4 / 2004$ \\
\hline '49FL' & 6/7-9/1997 & $5 / 31 / 1998$ & $5 / 28 / 1999$ & $5 / 12 / 2000$ & $6 / 22 / 2001$ & $4 / 5 / 2002$ & $3 / 20 / 2003$ & $6 / 4 / 2004$ \\
\hline '51FL' & $6 / 5 / 1997$ & 5/28-29/1998 & 5/26/1999 & $5 / 10 / 2000$ & $6 / 20 / 2001$ & $\begin{array}{l}4 / 3 / 2002 \\
9 / 12 / 2002\end{array}$ & $3 / 19 / 2003$ & $6 / 3 / 2004$ \\
\hline '75FL' & 6/6/1997 & $5 / 30 / 1998$ & 5/27/1999 & $5 / 11 / 2000$ & $6 / 21 / 2001$ & $\begin{array}{l}4 / 4 / 2002 \\
9 / 12 / 2002\end{array}$ & $3 / 19 / 2003$ & $6 / 3 / 2004$ \\
\hline '923_' & & $5 / 31 / 1998$ & $5 / 28 / 1999$ & $5 / 12 / 2000$ & $6 / 22 / 2001$ & $4 / 5 / 2002$ & $3 / 20 / 2003$ & $6 / 4 / 2004$ \\
\hline 'AIN2' & 6/7-9/1997 & $5 / 30 / 1998$ & 5/27/1999 & $5 / 11 / 2000$ & $6 / 21 / 2001$ & $\begin{array}{c}4 / 4,9 / 12-30 \\
11 / 1-4 / 2002\end{array}$ & & \\
\hline 'AINA' & $6 / 10 / 1997$ & 6/9-10/1998 & $5 / 14 / 1999$ & $5 / 13-15 / 2000$ & $6 / 23 / 2001$ & $\begin{array}{l}\text { 4/6/2002, } \\
10 / 8 / 2002\end{array}$ & & \\
\hline 'AKLA' & 1/28-30/1997 & $5 / 13 / 1998$ & 6/8-9/1999 & & $\begin{array}{c}6 / 12- \\
13 / 2001\end{array}$ & $7 / 26 / 2002$ & $4 / 17-18 / 2003$ & \\
\hline 'ALEA' & 6/4/1997 & $5 / 27 / 1998$ & $5 / 25 / 1999$ & $5 / 9 / 2000$ & $6 / 19 / 2001$ & $\begin{array}{l}4 / 2 / 2002 \\
9 / 12 / 2002\end{array}$ & $3 / 18 / 2003$ & $9 / 22-24 / 2004$ \\
\hline 'BRSH' & $6 / 4 / 1997$ & $5 / 27 / 1998$ & $5 / 25 / 1999$ & $5 / 9 / 2000$ & $6 / 19 / 2001$ & $4 / 3 / 2002$ & $3 / 18 / 2003$ & $6 / 2 / 2004$ \\
\hline 'ELEK' & $6 / 10 / 1997$ & $6 / 1-2 / 1998$ & $5 / 29 / 1999$ & $5 / 13-15 / 2000$ & $6 / 23 / 2001$ & $4 / 6 / 2002$ & $3 / 21 / 2003$ & $6 / 4 / 2004$ \\
\hline 'ELER' & 6/7-9/1997 & $6 / 1-2 / 1998$ & $7 / 10-12 / 1999$ & & & & & \\
\hline 'ENDR' & 6/5/1997 & $\begin{array}{l}5 / 27 / 1998 \\
6 / 18 / 1998\end{array}$ & $6 / 2 / 1999$ & & & & & \\
\hline 'HINA' & 1/28-30/1997 & $5 / 13 / 1998$ & 6/8-9/1999 & & $\begin{array}{c}6 / 12- \\
13 / 2001 \\
\end{array}$ & & $4 / 17 / 2003$ & \\
\hline 'HONM' & & $5 / 31 / 1998$ & $5 / 28 / 1999$ & $5 / 12 / 2000$ & $6 / 22 / 2001$ & $4 / 5 / 2002$ & $3 / 20 / 2003$ & $6 / 4 / 2004$ \\
\hline 'KFAS' & 6/10/1997 & $6 / 1-2 / 1998$ & $5 / 29 / 1999$ & $5 / 13-15 / 2000$ & $6 / 23 / 2001$ & $\begin{array}{c}4 / 6 / 2002,11 / 23- \\
24 / 2002 \\
\end{array}$ & $3 / 21 / 2003$ & $9 / 22-24 / 2004$ \\
\hline 'KFLT' & & 6/9-10/1998 & $5 / 14 / 1999$ & & & $7 / 24-25 / 2002$ & $3 / 11 / 2003$ & \\
\hline 'KULN' & $6 / 24-25 / 1997$ & 6/30-7/1/1998 & $6 / 29-30 / 1999$ & $7 / 1-3 / 2000$ & $\begin{array}{r}5 / 31- \\
6 / 1 / 2001\end{array}$ & & $10 / 10-12 / 2003$ & $12 / 21-22 / 2004$ \\
\hline
\end{tabular}




\begin{tabular}{|c|c|c|c|c|c|c|c|c|}
\hline Code & 1997 & 1998 & 1999 & 2000 & 2001 & 2002 & 2003 & 2004 \\
\hline 'KULU' & 6/10/1997 & $6 / 1-2 / 1998$ & $5 / 29 / 1999$ & $5 / 13-14 / 2000$ & $6 / 23 / 2001$ & $\begin{array}{c}4 / 6 / 2002,11 / 23- \\
24 / 2002\end{array}$ & $3 / 21 / 2003$ & $6 / 4 / 2004$ \\
\hline 'LACY' & $6 / 12-13 / 1997$ & $6 / 3-5 / 1998$ & $6 / 9-11 / 1999$ & $5 / 25-26 / 2000$ & $6 / 6-7 / 2001$ & $7 / 24-25 / 2002$ & $3 / 11-12 / 2003$ & $6 / 16-17 / 2004$ \\
\hline 'LKAO' & 6/4/1997 & & $5 / 25 / 1999$ & & & & & \\
\hline 'LUAK' & $4 / 29 / 1997$ & & & & & & $4 / 17-18 / 2003$ & \\
\hline 'ML1_' & 6/6/1997 & $5 / 30 / 1998$ & $5 / 27 / 1999$ & & & & & \\
\hline 'ML8_' & 6/10/1997 & $6 / 1-2 / 1998$ & $5 / 28 / 1999$ & $5 / 12 / 2000$ & $6 / 22 / 2001$ & $\begin{array}{c}4 / 6 / 2002,11 / 23- \\
24 / 2002 \\
\end{array}$ & $3 / 21 / 2003$ & \\
\hline 'MLCE' & 6/7-9/1997 & $5 / 30 / 1998$ & $5 / 27 / 1999$ & $5 / 11 / 2000$ & $6 / 21 / 2001$ & $4 / 4 / 2002$ & $3 / 21 / 2003$ & $6 / 3 / 2004$ \\
\hline 'MLO_' & 6/4-6/1997 & $6 / 4-5 / 1998$ & $5 / 26 / 1999$ & $5 / 24-25 / 2000$ & $\begin{array}{c}5 / 30- \\
6 / 1 / 2001 \\
\end{array}$ & $\begin{array}{l}7 / 23-24 / 2002 . \\
10 / 10-11 / 2002 \\
\end{array}$ & & \\
\hline 'MLST' & $\begin{array}{l}\text { 1/16-17/1997, } \\
2 / 10-12 / 1997\end{array}$ & & & & & & & \\
\hline 'MLSW' & & $5 / 30 / 1998$ & $5 / 26 / 1999$ & $5 / 10 / 2000$ & $6 / 20 / 2001$ & $\begin{array}{c}4 / 3 / 2002,11 / 23- \\
24 / 2002\end{array}$ & $3 / 19 / 2003$ & $6 / 3 / 2004$ \\
\hline 'MOAU' & & $5 / 13 / 1998$ & 4/28-29/1999 & & $\begin{array}{c}6 / 12- \\
13 / 2001 \\
\end{array}$ & $7 / 26 / 2002$ & $4 / 17-18 / 2003$ & \\
\hline 'MOKU' & $6 / 10 / 1997$ & $6 / 1-2 / 1998$ & $5 / 29 / 1999$ & $5 / 13-15 / 2000$ & $6 / 23 / 2001$ & $4 / 4 / 2002$ & $3 / 21 / 2003$ & $6 / 4 / 2004$ \\
\hline 'NAAL' & $4 / 29 / 1997$ & & 6/8-9/1999 & & $\begin{array}{c}6 / 13- \\
14 / 2001 \\
\end{array}$ & $7 / 26 / 2002$ & $3 / 26-27 / 2003$ & \\
\hline 'NOWR' & & $5 / 27 / 1998$ & $5 / 25 / 1999$ & $5 / 9 / 2000$ & $6 / 19 / 2001$ & $4 / 2 / 2002$ & $3 / 18 / 2003$ & $6 / 2 / 2004$ \\
\hline 'NWML' & $6 / 4 / 1997$ & $5 / 27 / 1998$ & $5 / 25 / 1999$ & $5 / 9 / 2000$ & $6 / 19 / 2001$ & $4 / 2 / 2002$ & $3 / 18 / 2003$ & $6 / 2 / 2004$ \\
\hline 'P103' & $6 / 12 / 1997$ & 6/4-5/1998 & 7/1-2/1999 & $5 / 24-25 / 2000$ & $\begin{array}{c}5 / 30- \\
31 / 2001\end{array}$ & $7 / 23-24 / 2002$ & $3 / 12-13 / 2003$ & $7 / 1-2 / 2004$ \\
\hline 'PLUU' & & $5 / 12-14 / 1998$ & $5 / 1-3 / 1999$ & & $6 / 6-7 / 2001$ & $7 / 25 / 2002$ & $3 / 26-27 / 2003$ & $6 / 16-17 / 2004$ \\
\hline 'POHO' & $4 / 29 / 1997$ & & 6/8-9/1999 & & $\begin{array}{c}7 / 10- \\
12 / 2001 \\
\end{array}$ & & $5 / 21-23 / 2003$ & $6 / 22-23 / 2004$ \\
\hline 'POUO' & $6 / 5 / 1997$ & 5/28-29/1998 & $5 / 26 / 1999$ & $5 / 10 / 2000$ & $6 / 20 / 2001$ & $4 / 3 / 2002$ & & $6 / 3 / 2004$ \\
\hline 'REDC' & 6/7-9/1997 & $5 / 31 / 1998$ & $5 / 28 / 1999$ & $5 / 11 / 2000$ & $6 / 21 / 2001$ & $\begin{array}{l}4 / 5 / 2002 \\
9 / 12 / 2002\end{array}$ & $3 / 20 / 2003$ & $6 / 4 / 2004$ \\
\hline 'SELH' & $6 / 7-9 / 1997$ & $5 / 31 / 1998$ & $5 / 27 / 1999$ & $5 / 11 / 2000$ & $6 / 21 / 2001$ & $4 / 4 / 2002$ & $3 / 20 / 2003$ & $6 / 3 / 2004$ \\
\hline 'V134' & $6 / 5 / 1997$ & $5 / 28-29 / 1998$ & $5 / 26 / 1999$ & $5 / 10 / 2000$ & $6 / 20 / 2001$ & $4 / 3 / 2002$ & $3 / 19 / 2003$ & $6 / 3 / 2004$ \\
\hline 'VANC' & $6 / 12 / 1997$ & 6/4-5/1998 & $5 / 26 / 1999$ & $5 / 24-25 / 2000$ & $\begin{array}{c}5 / 30- \\
6 / 1 / 2001\end{array}$ & $7 / 23-24 / 2002$ & $3 / 12-13 / 2003$ & $7 / 1-2 / 2004$ \\
\hline
\end{tabular}




\begin{tabular}{|c|c|c|c|c|c|c|c|c|}
\hline Code & 1997 & 1998 & 1999 & 2000 & 2001 & 2002 & 2003 & 2004 \\
\hline 'VENT' & $6 / 4 / 1997$ & $6 / 18 / 1998$ & $5 / 25 / 1999$ & $5 / 9 / 2000$ & $6 / 19 / 2001$ & $4 / 2 / 2002$ & $3 / 27-28 / 2003$ & $6 / 2 / 2004$ \\
\hline 'VO92' & $6 / 6 / 1997$ & $5 / 30 / 1998$ & $5 / 27 / 1999$ & $5 / 10 / 2000$ & $6 / 20 / 2001$ & $4 / 4 / 2002$ & $3 / 18 / 2003$ & $6 / 2 / 2004$ \\
\hline 'WAIH' & & $5 / 13 / 1998$ & 4/28-29/1999 & & $\begin{array}{c}6 / 12- \\
13 / 2001 \\
\end{array}$ & $7 / 26 / 2002$ & $4 / 17-18 / 2003$ & \\
\hline 'WMLS' & 6/6/1997 & $5 / 28 / 1998$ & $5 / 26 / 1999$ & $5 / 10 / 2000$ & $6 / 20 / 2001$ & $4 / 2 / 2002$ & $3 / 19 / 2003$ & $6 / 3 / 2004$ \\
\hline \multicolumn{9}{|c|}{ WEST MAUNA LOA } \\
\hline 'HONO' & & & $6 / 17 / 1999$ & & & & & \\
\hline 'HONR' & $4 / 29 / 1997$ & & $6 / 17 / 1999$ & & $7 / 11 / 2001$ & & $5 / 20-21 / 2003$ & \\
\hline KEAL & $4 / 30-5-1 / 1997$ & & 6/16/1999 & & $\begin{array}{c}7 / 10- \\
12 / 2001 \\
\end{array}$ & & $5 / 22-23 / 2003$ & \\
\hline 'KEEl' & 4/30/1997 & & $6 / 17 / 1999$ & & $7 / 11 / 2001$ & & $5 / 20,22 / 2003$ & \\
\hline 'KNKI' & $4 / 30 / 1997$ & & $6 / 16 / 1999$ & & $7 / 11 / 2001$ & & $5 / 22 / 2003$ & \\
\hline 'KRCN' & $4 / 30 / 1997$ & & $6 / 16 / 1999$ & & $7 / 11 / 2001$ & & $5 / 20,22 / 2003$ & \\
\hline 'MILO' & $4 / 29 / 1997$ & & $6 / 15 / 1999$ & & $7 / 10 / 2001$ & & $5 / 21 / 2003$ & \\
\hline 'YEHP' & $4 / 29 / 1997$ & & 6/16-17/1999 & & $7 / 6 / 2001$ & & $5 / 22-23 / 2003$ & \\
\hline 'YHP3' & & & & & $7 / 6 / 2001$ & & $5 / 22 / 2003$ & \\
\hline
\end{tabular}




\begin{tabular}{|c|c|c|c|c|c|c|c|c|}
\hline Code & 1997 & 1998 & 1999 & 2000 & 2001 & 2002 & 2003 & 2004 \\
\hline \multicolumn{9}{|c|}{ HUALALAI } \\
\hline '1800' & & 4/2/1998 & & & $\begin{array}{c}8 / 8- \\
9 / 2001\end{array}$ & & $\begin{array}{c}6 / 18- \\
19 / 2003 \\
\end{array}$ & \\
\hline 'HALE' & & $4 / 2 / 1998$ & & & $8 / 9 / 2001$ & & $6 / 19 / 2003$ & \\
\hline 'HOLU' & $\begin{array}{c}5 / 1- \\
2 / 1997 \\
\end{array}$ & & & & $\begin{array}{c}\text { 8/8- } \\
9 / 2001 \\
\end{array}$ & & $6 / 18 / 2003$ & \\
\hline 'IKAA' & & 4/1/1998 & & & $8 / 8 / 2001$ & & $6 / 18 / 2003$ & \\
\hline 'JUDD' & & 4/1/1998 & & & $8 / 8 / 2001$ & & & \\
\hline 'KEHU' & & 4/2/1998 & & & $\begin{array}{c}\text { 8/8- } \\
9 / 2001 \\
\end{array}$ & & $\begin{array}{c}6 / 18- \\
19 / 2003 \\
\end{array}$ & \\
\hline 'KIPA' & & $4 / 3 / 1998$ & & & $8 / 9 / 2001$ & & $6 / 19 / 2003$ & \\
\hline 'LOHA' & & & & & $8 / 8 / 2001$ & & $6 / 18 / 2003$ & \\
\hline 'MAAU' & & 4/1/1998 & & & $8 / 9 / 2001$ & & $6 / 19 / 2003$ & \\
\hline 'MALE' & $5 / 2 / 1997$ & $4 / 3 / 1998$ & & & $8 / 10 / 2001$ & & $6 / 20 / 2003$ & \\
\hline 'MORC' & & 4/1/1998 & & & $8 / 9 / 2001$ & & $6 / 20 / 2003$ & \\
\hline 'NCAB' & & & & & $8 / 10 / 2001$ & & $6 / 20 / 2003$ & \\
\hline 'NOHO' & & & & & $8 / 8 / 2001$ & & $6 / 19 / 2003$ & \\
\hline 'WAIA' & $5 / 2 / 1997$ & 4/3/1998 & & & & & $6 / 18 / 2003$ & \\
\hline \multicolumn{9}{|l|}{ MAUI } \\
\hline 'HAIL' & $7 / 25 / 1997$ & & & & & & & \\
\hline 'HANA' & 7/24/1997 & & & & & & & \\
\hline 'HKHI' & $\begin{array}{c}7 / 23- \\
25 / 1997 \\
\end{array}$ & & & & & & & \\
\hline KHUA & $7 / 25 / 1997$ & & & & & & & \\
\hline 'KNAE' & 7/24/1997 & & & & & & & \\
\hline 'KOLE' & $\begin{array}{c}7 / 22- \\
25 / 1997\end{array}$ & $\begin{array}{c}4 / 21- \\
25 / 1998\end{array}$ & & & & & & \\
\hline 'LEIE' & $7 / 24 / 1997$ & & & & & & & \\
\hline 'LOOK' & $7 / 22 / 1997$ & & & & & & & \\
\hline 'LUAL' & $7 / 22 / 1997$ & & & & & & & \\
\hline 'NIAN' & $\begin{array}{c}7 / 22- \\
23 / 1997\end{array}$ & & & & & & & \\
\hline 'POLI' & $7 / 25 / 1997$ & & & & & & & \\
\hline 'PUUI' & $\begin{array}{c}7 / 22- \\
23 / 1997\end{array}$ & & & & & & & \\
\hline \multicolumn{9}{|c|}{ MISCELLANEOUS } \\
\hline 'BLDR' & $\begin{array}{r}1 / 16-17 \\
6 / 12 \\
9 / 11- \\
12 / 1997 \\
\end{array}$ & $\begin{array}{r}6 / 5 / 1998 \\
7 / 9-10 / 1998\end{array}$ & $\begin{array}{r}5 / 27 / 1999, \\
7 / 1- \\
2 / 1999\end{array}$ & $\begin{array}{r}5 / 24- \\
25 / 2000\end{array}$ & $\begin{array}{r}5 / 30- \\
31 / 2001\end{array}$ & $\begin{array}{r}7 / 2- \\
243 / 2002 \\
\end{array}$ & $\begin{array}{r}3 / 12- \\
13 / 2003 \\
\end{array}$ & $7 / 1-2 / 2004$ \\
\hline 'HP7 ' & $\begin{array}{c}3 / 19 / 1997 \\
6 / 24- \\
25 / 1997\end{array}$ & $\begin{array}{c}6 / 30- \\
7 / 1 / 1998\end{array}$ & $\begin{array}{c}\text { 6/16- } \\
17 / 1999\end{array}$ & $\begin{array}{c}6 / 26- \\
27 / 2000\end{array}$ & $\begin{array}{c}7 / 10- \\
12 / 2001\end{array}$ & $\begin{array}{c}7 / 15- \\
17 / / 2002\end{array}$ & $\begin{array}{c}9 / 25- \\
26 / 2003\end{array}$ & $\begin{array}{c}12-16- \\
17 / 2004\end{array}$ \\
\hline KALK' & $\begin{array}{l}4 / 29-5- \\
2 / 1997\end{array}$ & 4/1-3/1998 & $\begin{array}{c}6 / 16- \\
17 / 1999\end{array}$ & & $\begin{array}{c}7 / 10- \\
12 / 2001\end{array}$ & & $5 / 21 / 2003$ & \\
\hline 'MKEA' & $\begin{array}{c}6 / 24- \\
25 / 1997 \\
9 / 11- \\
12 / 1997\end{array}$ & $\begin{array}{c}\text { 7/16- } \\
17 / 1998\end{array}$ & & & & & & \\
\hline 'RLYM' & $\begin{array}{r}6 / 23- \\
24 / 1997 \\
\end{array}$ & 6/25,30/1998 & & & & & & \\
\hline
\end{tabular}


Table 5a. 1997-2002 continuous station velocities, in meters

\begin{tabular}{|c|c|c|c|c|c|c|}
\hline Station & East & North & Up & N sigma & E sigma & U sigma \\
\hline \multicolumn{7}{|c|}{ Kilauea: July 011997 to Dec 012001} \\
\hline AHUP & 0.0186 & -0.0125 & -0.0489 & 0.0002 & 0.0002 & 0.0009 \\
\hline GOPM & 0.0285 & -0.0624 & 0.0136 & 0.0002 & 0.0002 & 0.0008 \\
\hline HALP & 0.0068 & -0.0201 & 0.0008 & 0.0010 & 0.0008 & 0.0035 \\
\hline HILO & 0.0008 & 0.0017 & 0.0022 & 0.0004 & 0.0003 & 0.0011 \\
\hline HOLE & 0.0352 & -0.0549 & 0.0200 & 0.0003 & 0.0003 & 0.0011 \\
\hline KAEP & 0.0351 & -0.0595 & 0.0193 & 0.0003 & 0.0002 & 0.0008 \\
\hline KOSM & 0.0384 & -0.0173 & -0.0343 & 0.0003 & 0.0002 & 0.0010 \\
\hline KTPM & 0.0313 & -0.0367 & 0.0119 & 0.0002 & 0.0002 & 0.0008 \\
\hline MANE & 0.0213 & -0.0226 & -0.0044 & 0.0003 & 0.0002 & 0.0010 \\
\hline MLPM & 0.0033 & 0.0001 & 0.0008 & 0.0004 & 0.0004 & 0.0012 \\
\hline NUPM & 0.0012 & -0.0287 & -0.0146 & 0.0002 & 0.0002 & 0.0008 \\
\hline PGF1 & 0.0148 & -0.0209 & 0.0077 & 0.0003 & 0.0002 & 0.0008 \\
\hline PGF2 & 0.0354 & -0.0657 & 0.0156 & 0.0003 & 0.0002 & 0.0008 \\
\hline PGF3 & 0.0249 & -0.0557 & 0.0162 & 0.0003 & 0.0002 & 0.0009 \\
\hline PGF4 & 0.0260 & -0.0566 & 0.0160 & 0.0003 & 0.0002 & 0.0008 \\
\hline PGF5 & 0.0181 & -0.0374 & 0.0128 & 0.0002 & 0.0002 & 0.0008 \\
\hline PGF6 & 0.0133 & -0.0252 & 0.0089 & 0.0003 & 0.0002 & 0.0008 \\
\hline UWEV & 0.0087 & -0.0151 & -0.0096 & 0.0002 & 0.0002 & 0.0009 \\
\hline WAOP & 0.0016 & -0.0105 & -0.0030 & 0.0010 & 0.0008 & 0.0034 \\
\hline \multicolumn{7}{|c|}{ Mauna Loa: Jan 011997 to May 012002} \\
\hline ELEP & 0.0068 & -0.0057 & -0.0022 & 0.0003 & 0.0003 & 0.0011 \\
\hline MLSP & 0.0056 & -0.0034 & -0.0119 & 0.0004 & 0.0003 & 0.0013 \\
\hline MOKP & 0.0090 & -0.0077 & -0.0077 & 0.0003 & 0.0003 & 0.0012 \\
\hline \multicolumn{7}{|c|}{ Mauna Kea velocity removed: this estimate from 1997 through 2004} \\
\hline MKPM & -0.0632 & $0.0333-$ & 00168 & 0.00000 & 0.00001 & 0.00006 \\
\hline
\end{tabular}


Table 5b. 2002-2004 continuous station velocities, in meters

\begin{tabular}{|l|r|r|r|r|r|r|}
\hline \multicolumn{1}{|c|}{ Station } & \multicolumn{1}{|c|}{ East } & \multicolumn{1}{c|}{ North } & \multicolumn{1}{c|}{ Up } & N sigma & E sigma & U sigma \\
\hline Kilauea: Dec 012001 to Dec 31 2004 & & & \\
\hline AHUP & 0.0158 & -0.0281 & -0.0242 & 0.0004 & 0.0004 & 0.0016 \\
\hline GOPM & 0.0211 & -0.0471 & 0.0130 & 0.0005 & 0.0004 & 0.0017 \\
\hline HALR & 0.0157 & -0.0300 & 0.0222 & 0.0009 & 0.0008 & 0.0033 \\
\hline HILO & -0.0016 & 0.0012 & 0.0020 & 0.0004 & 0.0004 & 0.0014 \\
\hline HOLE & 0.0284 & -0.0522 & 0.0215 & 0.0005 & 0.0004 & 0.0018 \\
\hline KAEP & 0.0283 & -0.0483 & 0.0169 & 0.0004 & 0.0004 & 0.0015 \\
\hline KOSM & 0.0260 & -0.0204 & -0.0241 & 0.0004 & 0.0004 & 0.0015 \\
\hline KTPM & 0.0193 & -0.0387 & 0.0173 & 0.0004 & 0.0003 & 0.0015 \\
\hline MANE & 0.0152 & -0.0284 & -0.0021 & 0.0004 & 0.0004 & 0.0017 \\
\hline MLPM & 0.0014 & -0.0004 & 0.0011 & 0.0004 & 0.0004 & 0.0020 \\
\hline NUPM & -0.0026 & -0.0132 & 0.0009 & 0.0004 & 0.0003 & 0.0014 \\
\hline PGF1 & 0.0101 & -0.0229 & 0.0055 & 0.0004 & 0.0004 & 0.0015 \\
\hline PGF2 & 0.0219 & -0.0478 & 0.0166 & 0.0005 & 0.0004 & 0.0016 \\
\hline PGF3 & 0.0159 & -0.0474 & 0.0170 & 0.0004 & 0.0004 & 0.0015 \\
\hline PGF4 & 0.0158 & -0.0472 & 0.0166 & 0.0004 & 0.0004 & 0.0016 \\
\hline PGF5 & 0.0117 & -0.0373 & 0.0061 & 0.0007 & 0.0006 & 0.0024 \\
\hline PGF6 & 0.0084 & -0.0276 & 0.0063 & 0.0005 & 0.0004 & 0.0016 \\
\hline UWEV & -0.0054 & 0.0019 & 0.0056 & 0.0004 & 0.0003 & 0.0014 \\
\hline WAOP & -0.0030 & 0.0038 & 0.0093 & 0.0005 & 0.0004 & 0.0018 \\
\hline Mauna Loa: May 012002 to Dec 31 2004 & & & \\
\hline ELEP & 0.0304 & -0.0111 & 0.0087 & 0.0005 & 0.0004 & 0.0017 \\
\hline MLSP & -0.0059 & -0.0111 & 0.0261 & 0.0005 & 0.0004 & 0.0016 \\
\hline MOKP & -0.0089 & 0.0059 & 0.0187 & 0.0005 & 0.0004 & 0.0017 \\
\hline AINP & 0.0148 & -0.0111 & 0.0009 & 0.0008 & 0.0007 & 0.0029 \\
\hline PAT2 & 0.0317 & -0.0307 & 0.0293 & 0.0012 & 0.0011 & 0.0051 \\
\hline STEP & -0.0017 & 0.0000 & 0.0050 & 0.0007 & 0.0007 & 0.0029 \\
\hline Mauna Kea velocity removed: this estimate from 1997 through 2004 & \\
\hline MKPM & -0.0632 & 0.0333 & -0.00168 & 0.00000 & 0.00001 & 0.00006 \\
\hline
\end{tabular}


AHUP
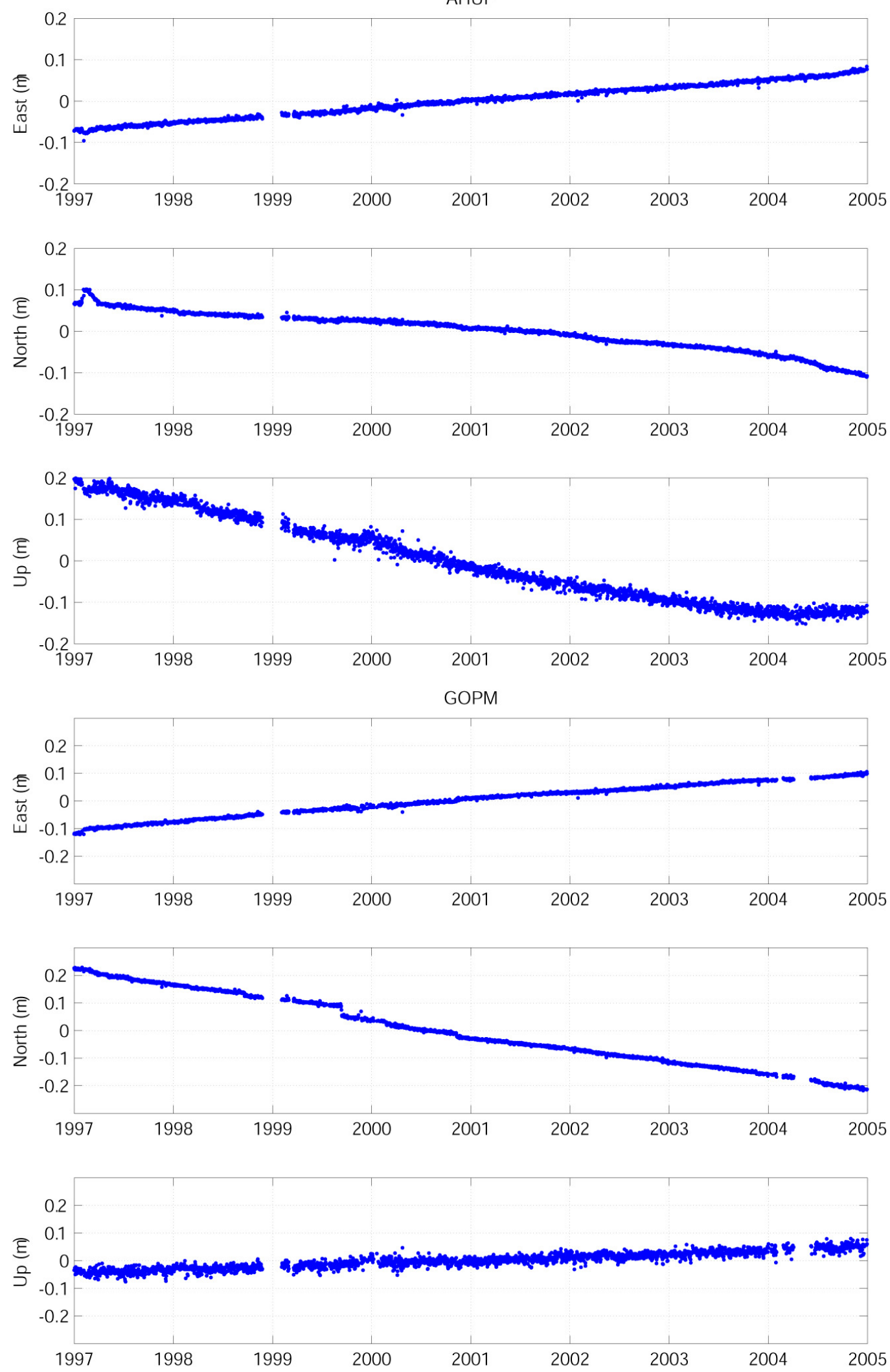

Figure 5a. Time series of continuously recording sites AHUP, GOPM. 
HALP
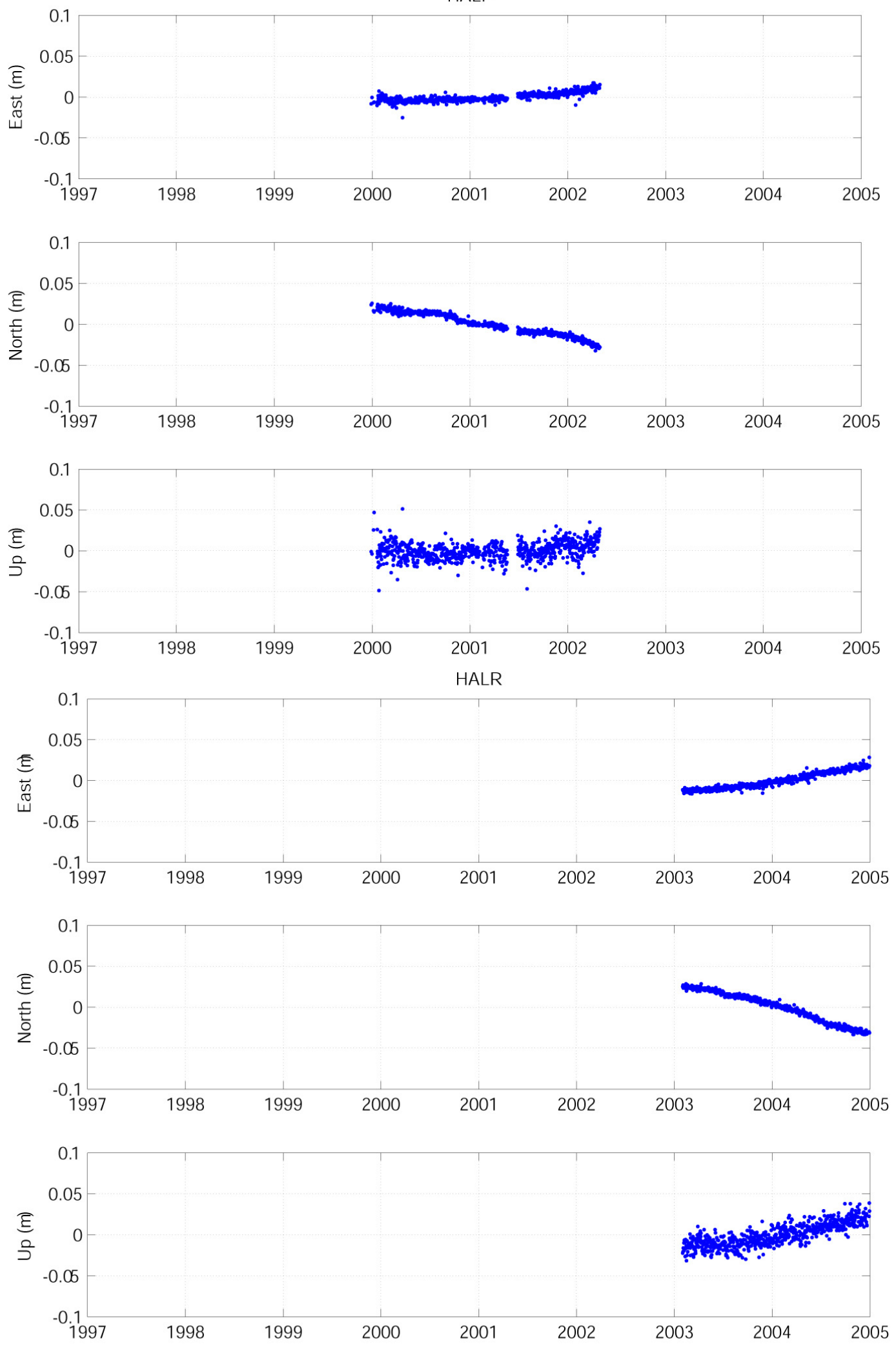

Figure $5 \mathrm{~b}$. Time series of continuously recording sites HALP, HALR. 
HILO
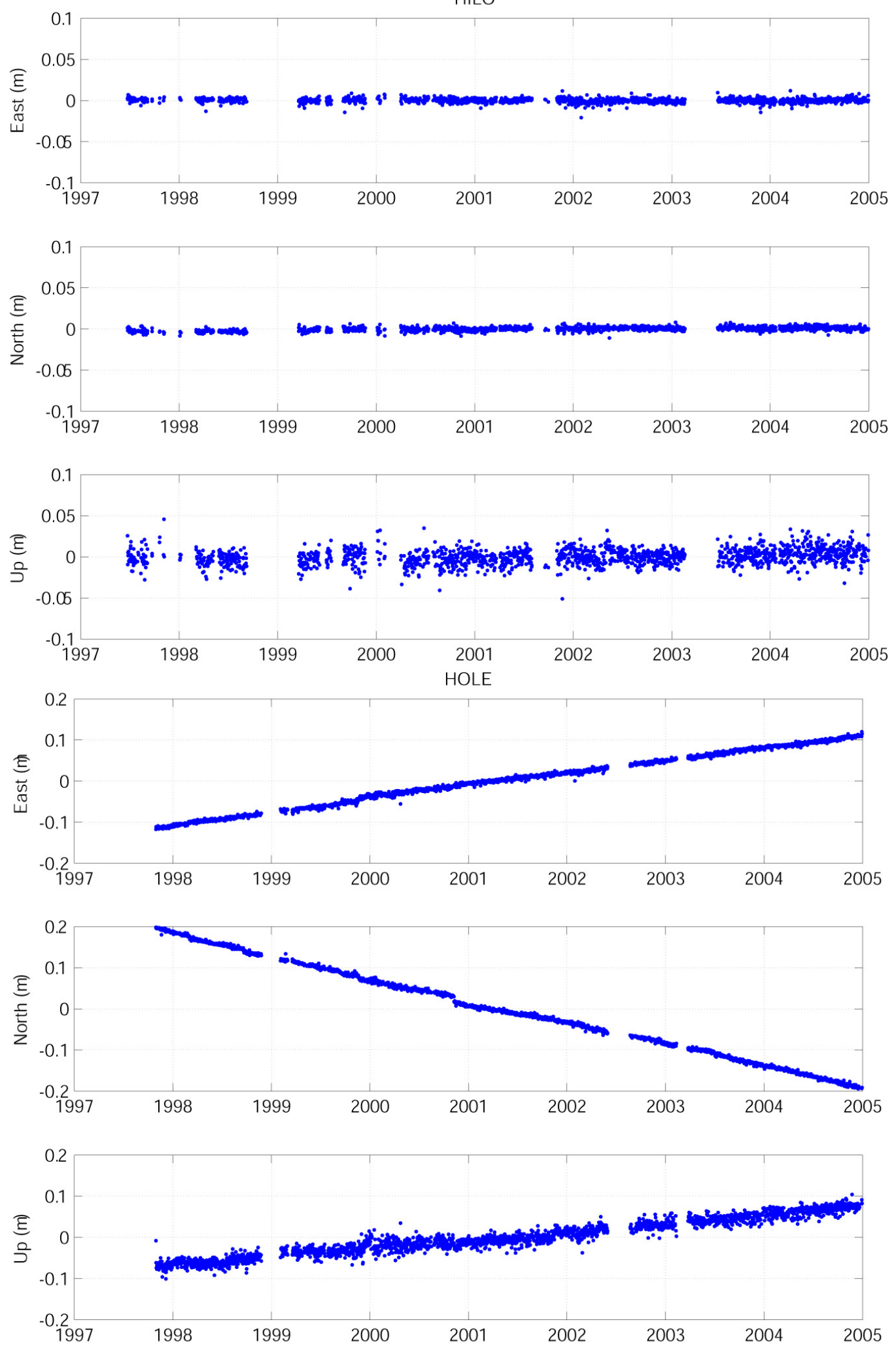

Figure 5c. Time series of continuously recording sites HILO, HOLE. 

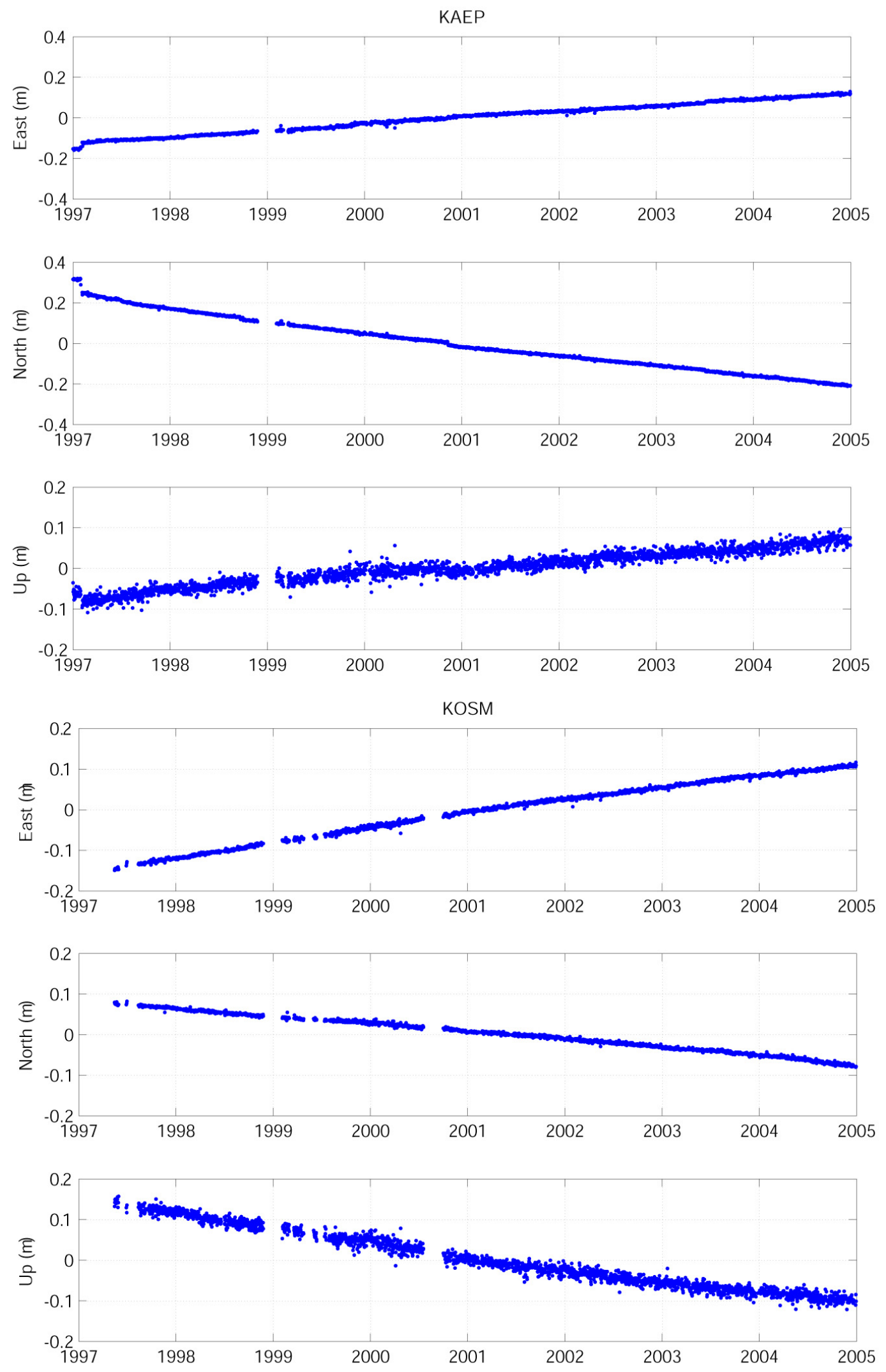

Figure $5 \mathrm{~d}$. Time series of continuously recording sites KAEP, KOSM. 

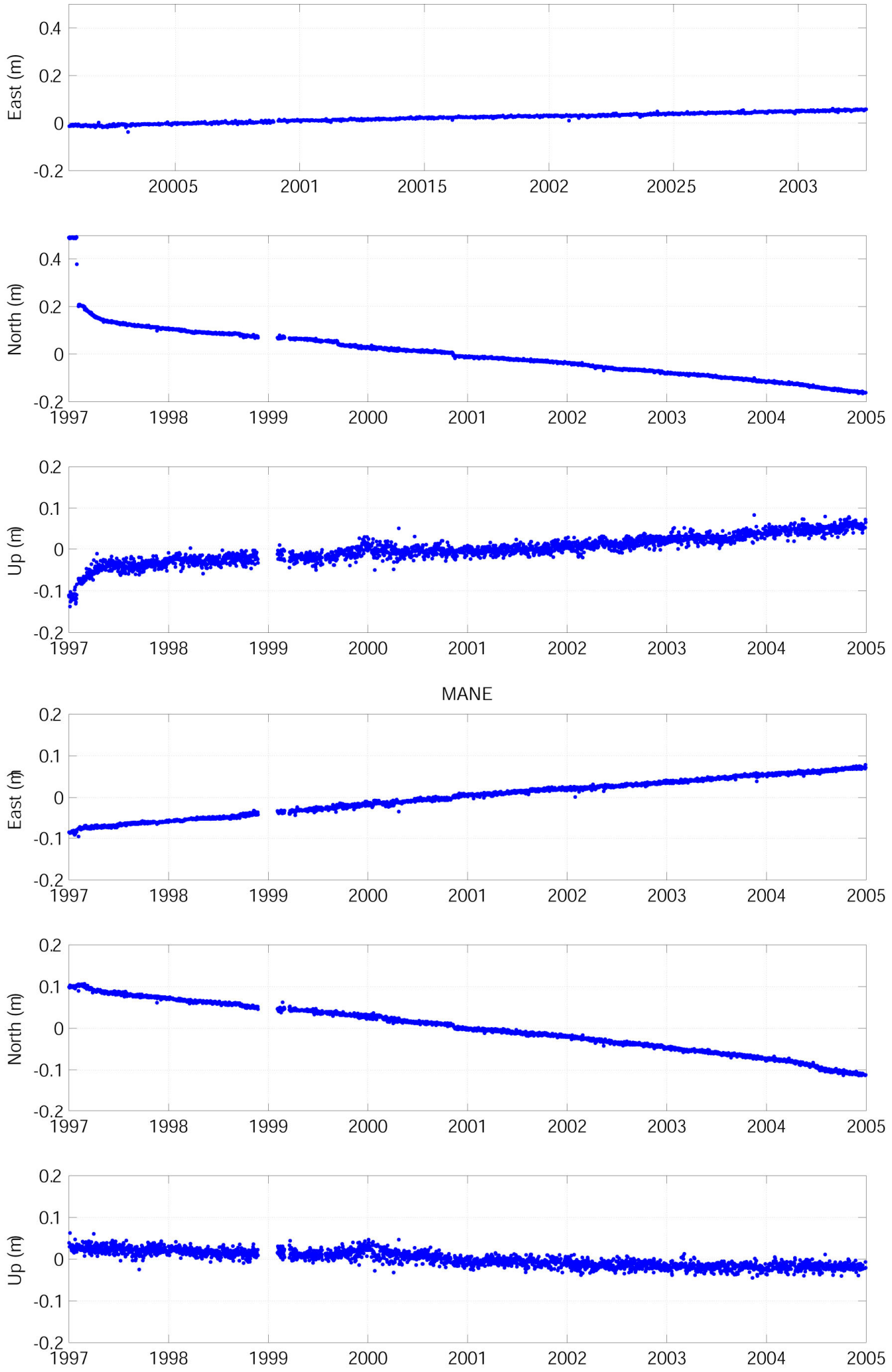

Figure $5 \mathrm{e}$. Time series of continuously recording sites KTPM, MANE. 

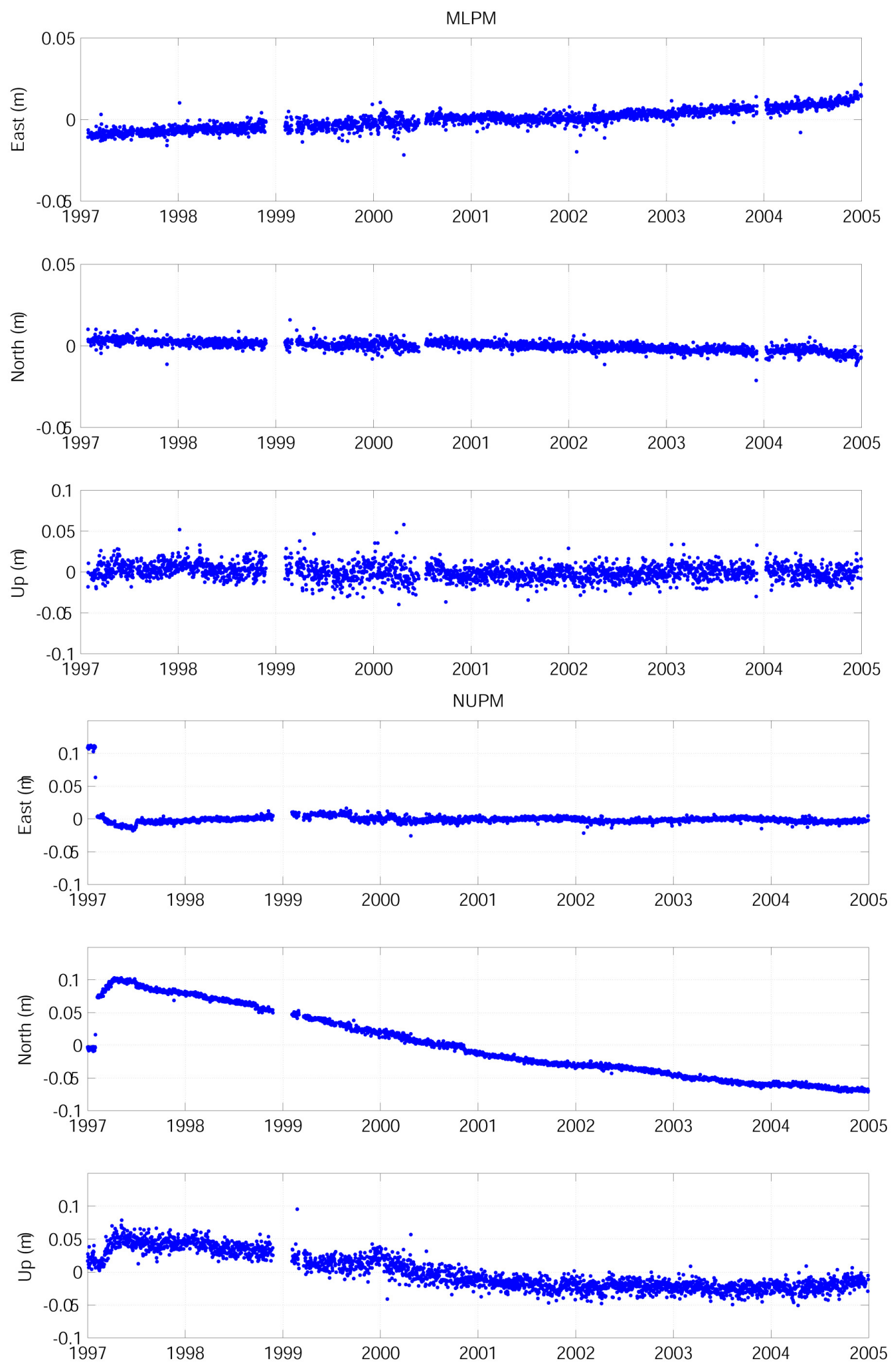

Figure $5 f$. Time series of continuously recording sites MLPM, NUPM. 

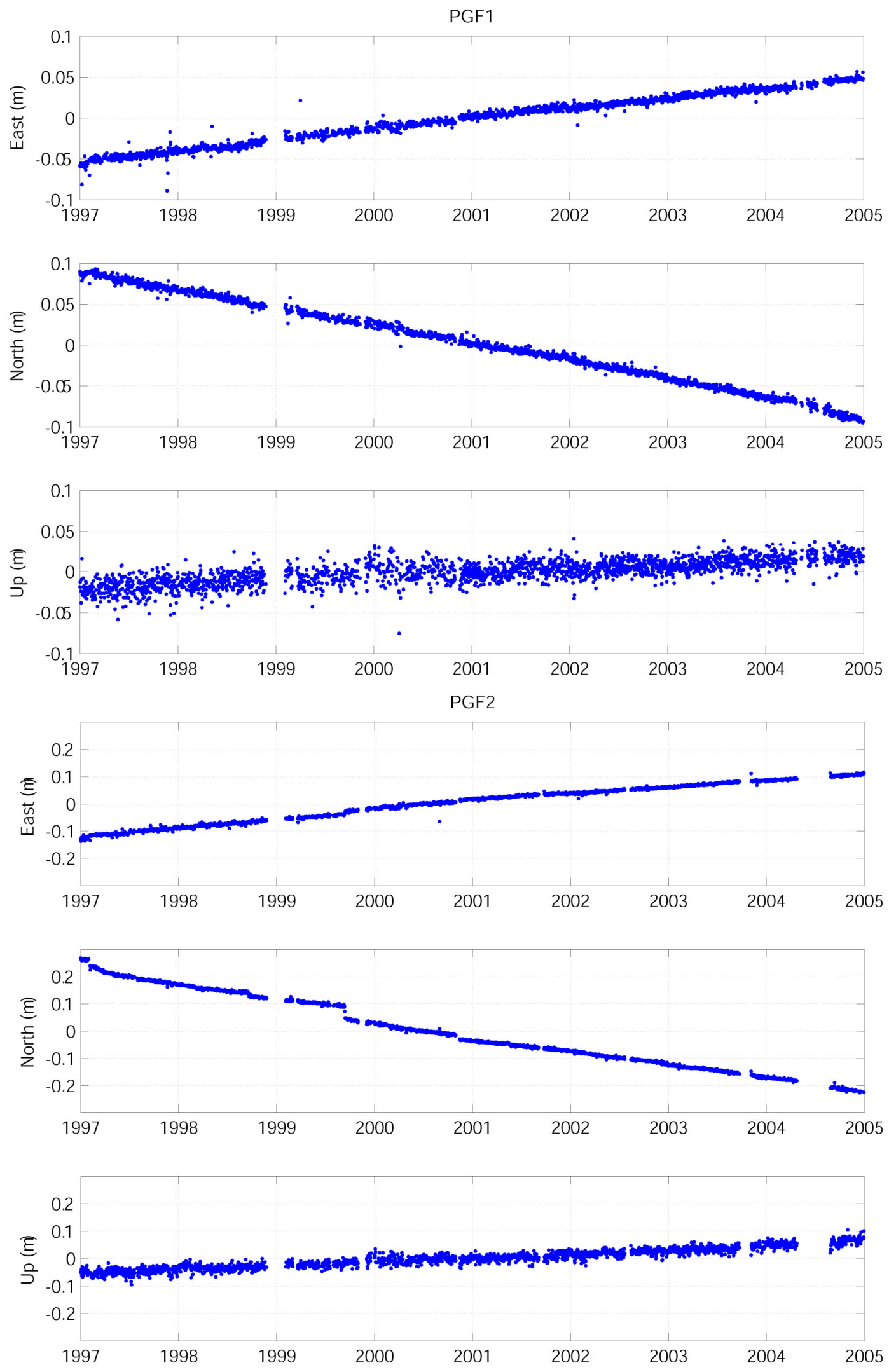

Figure $5 \mathrm{~g}$. Time series of continuously recording sites PGF1, PGF2. 
PGF3
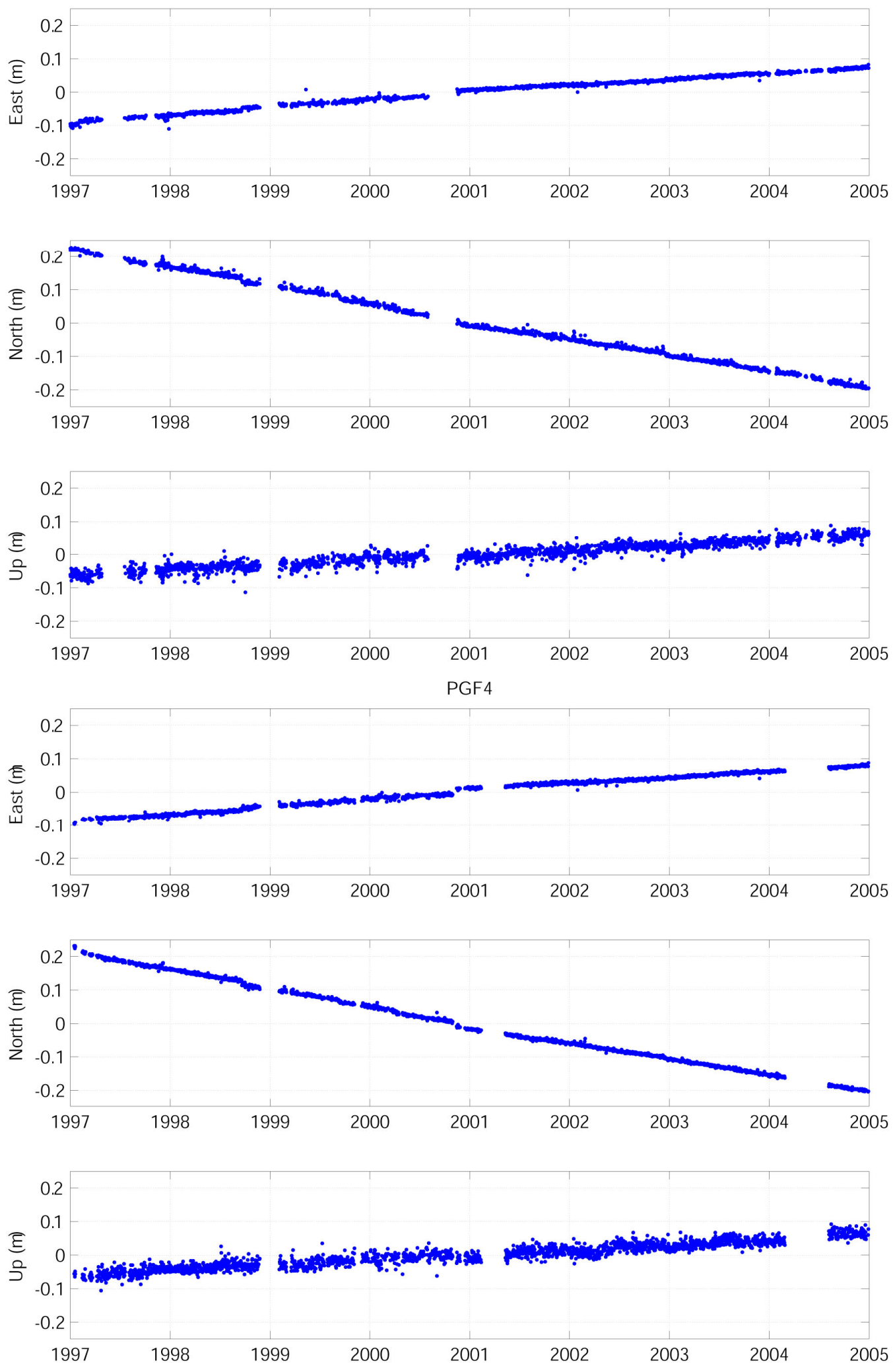

Figure 5h. Time series of continuously recording sites PGF3, PGF4. 

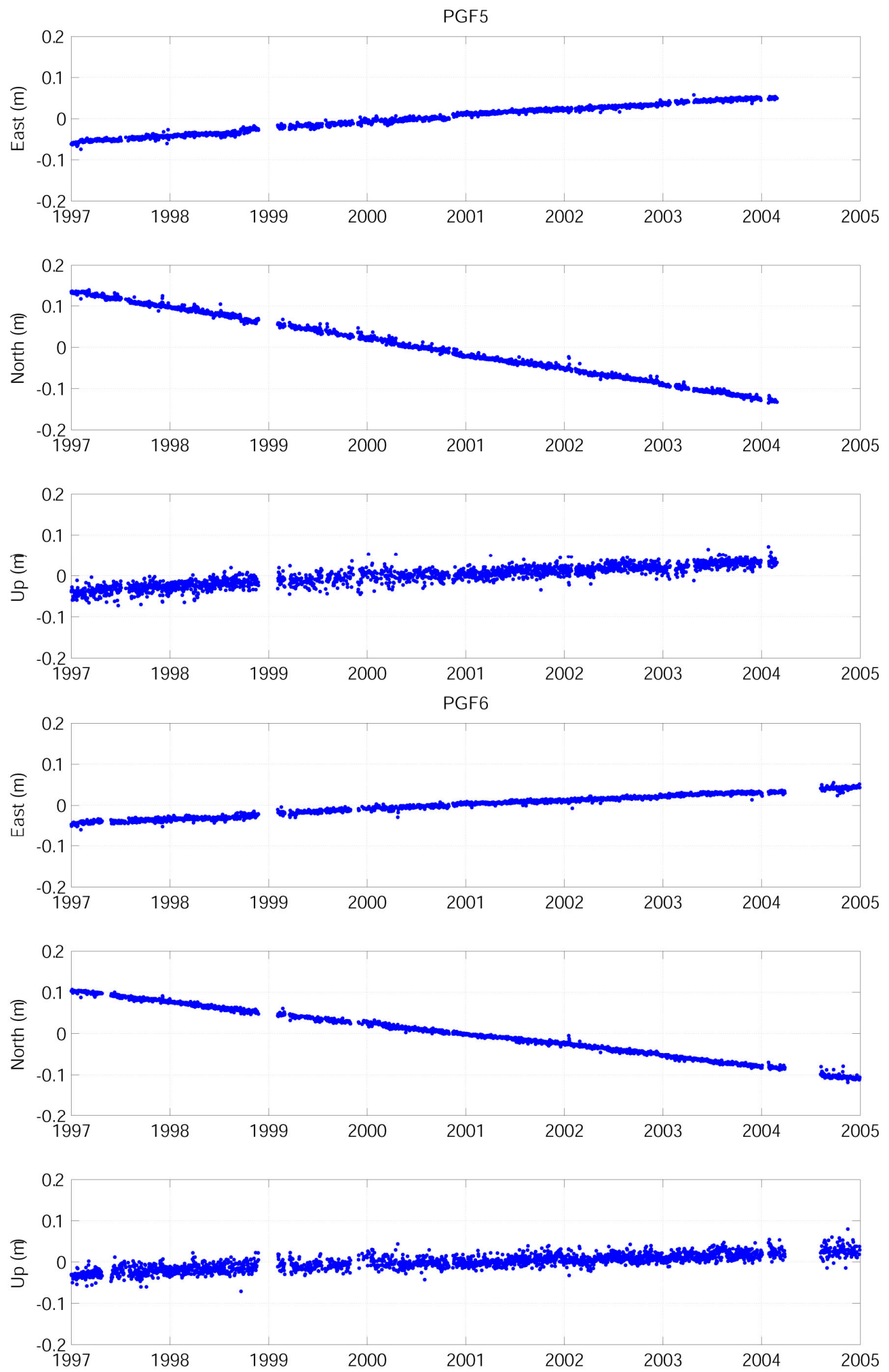

Figure 5i. Time series of continuously recording sites PGF5, PGF6. 

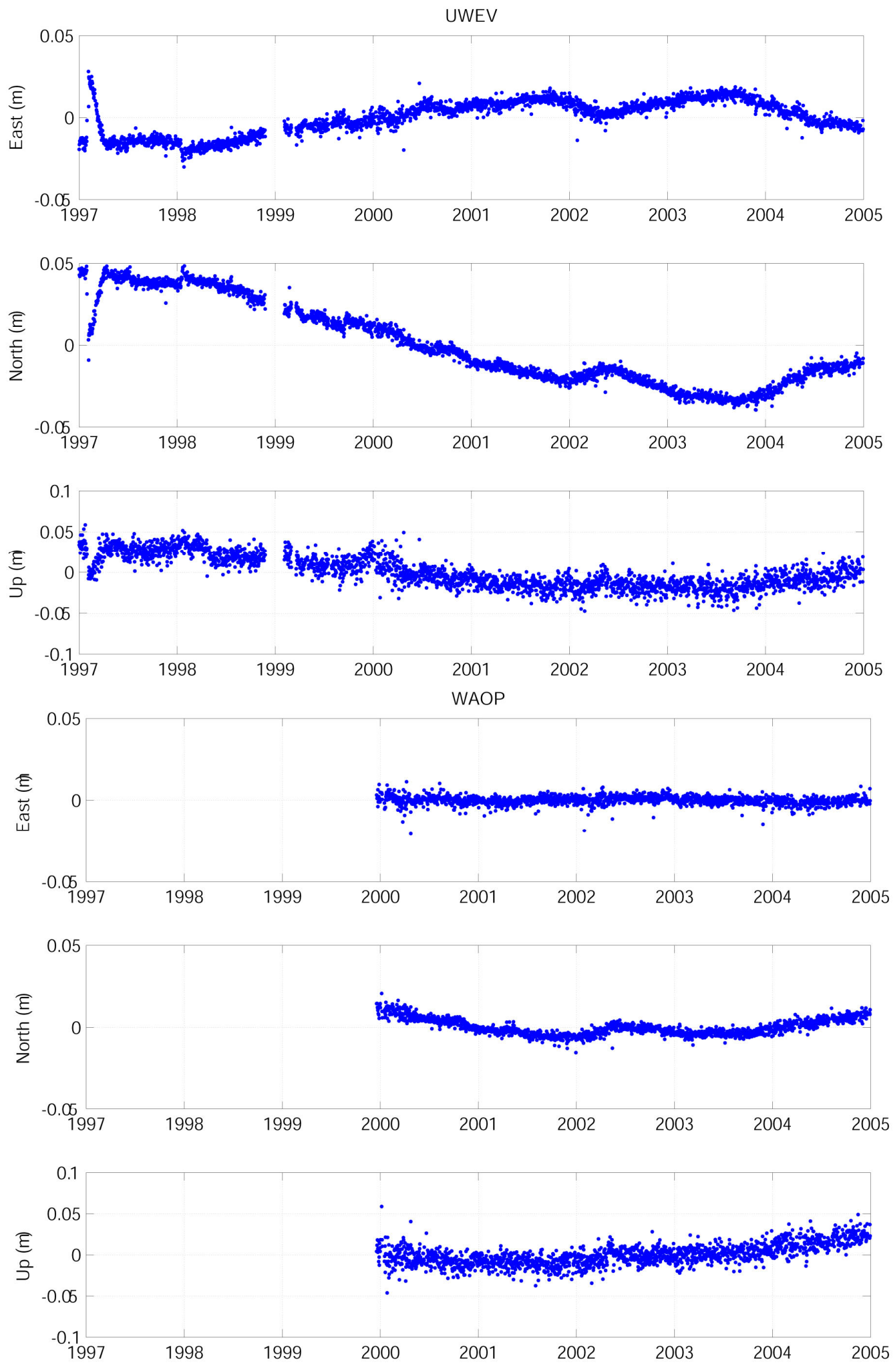

Figure 5j. Time series of continuously recording sites UWEV, WAOP. 

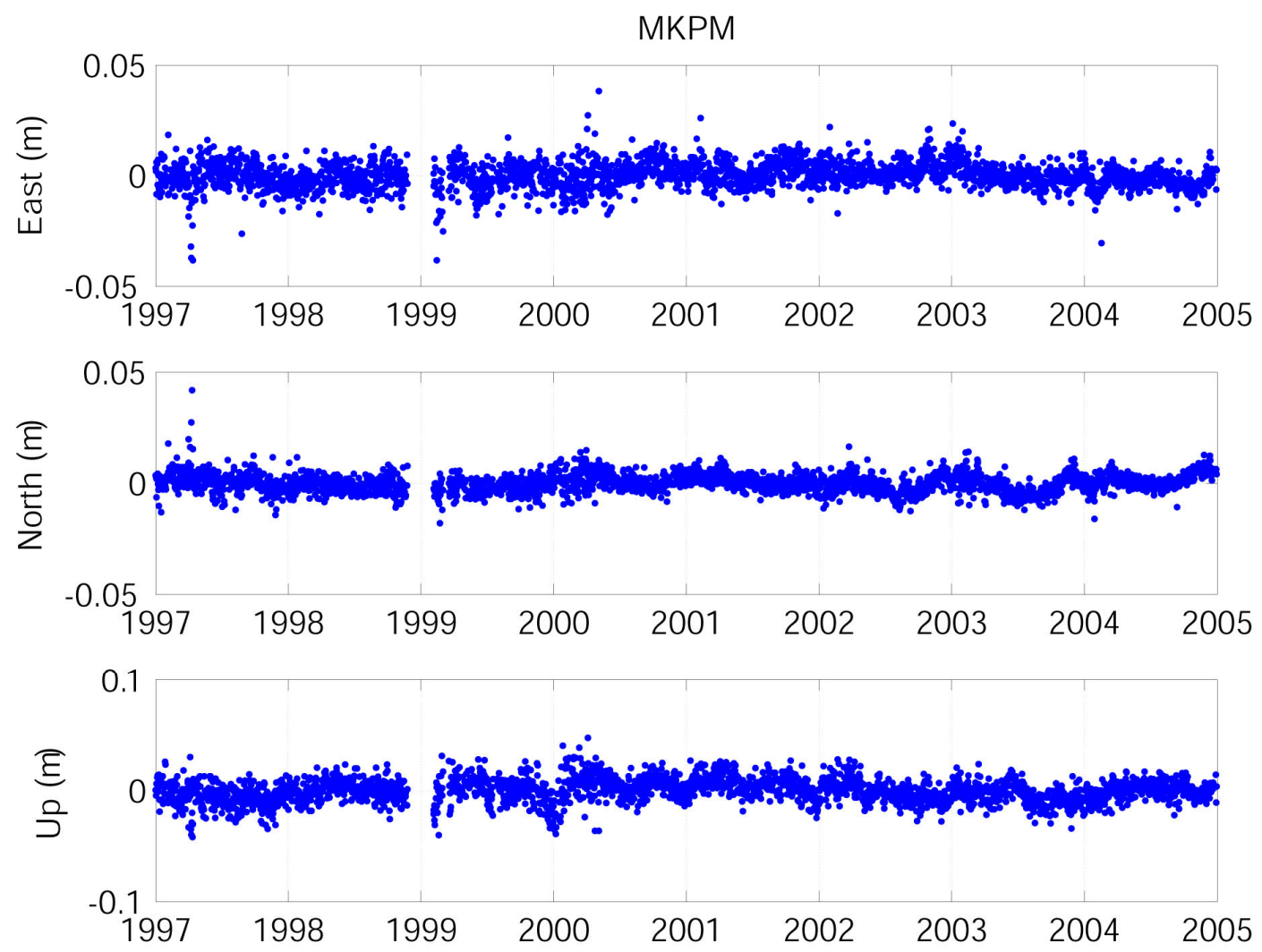

Figure $5 \mathrm{k}$. Time series of continuously recording site MKPM. 

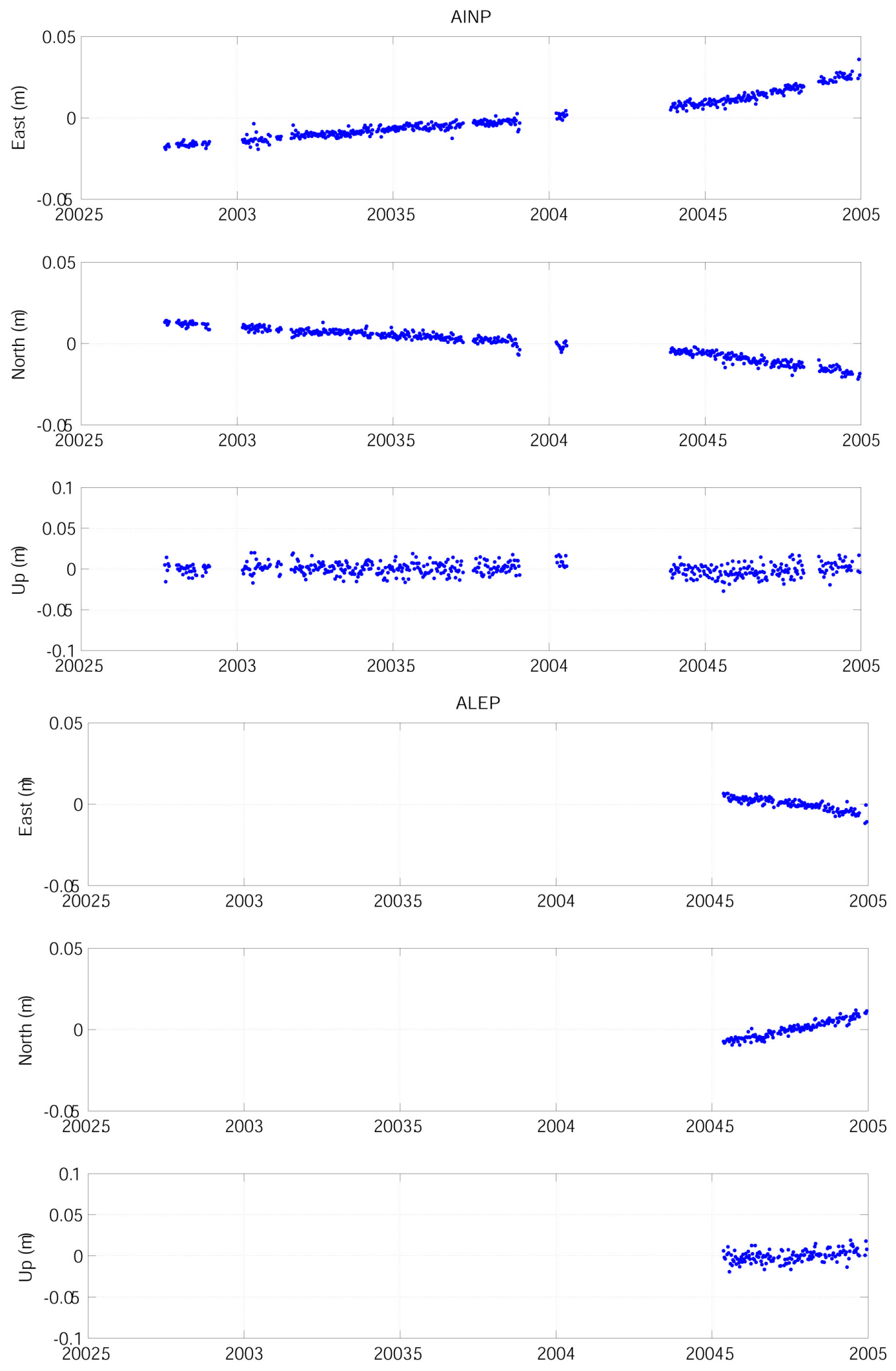

Figure 5I. Time series of continuously recording sites AINP, ALEP. 
BLBP
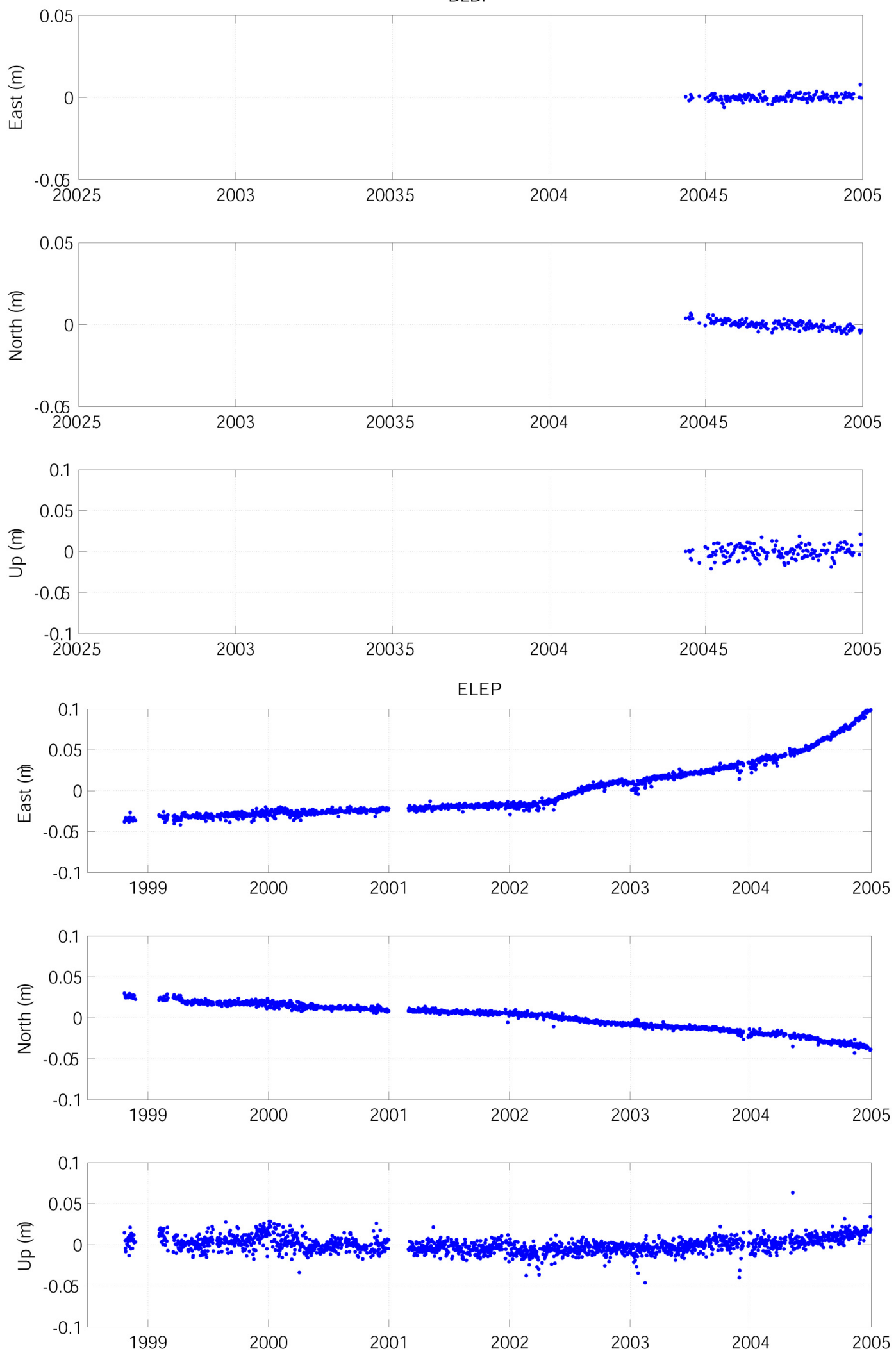

Figure $5 \mathrm{~m}$. Time series of continuously recording sites BLBP, ELEP. 

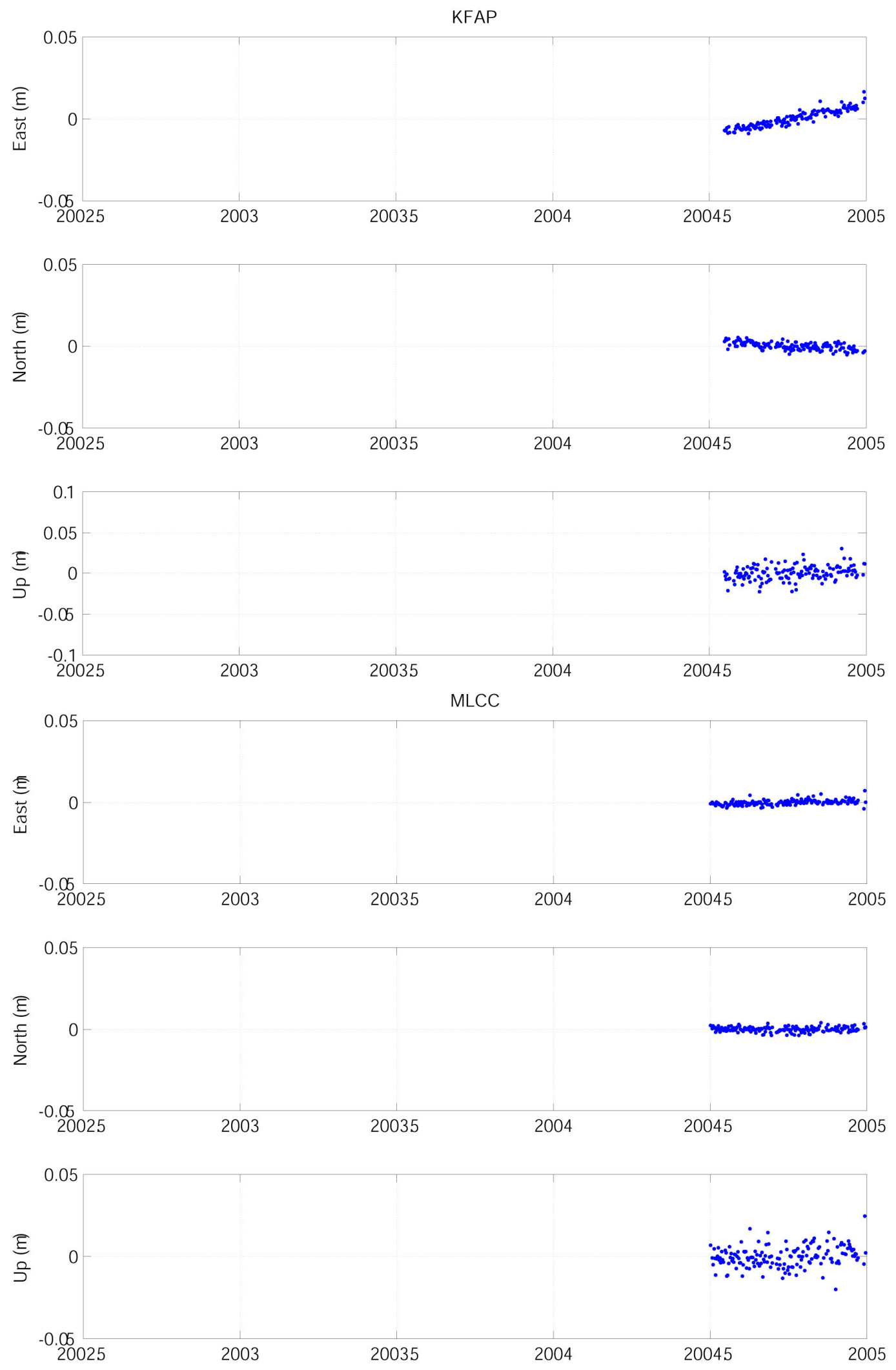

Figure $5 \mathrm{n}$. Time series of continuously recording sites KFAP, MLCC. 

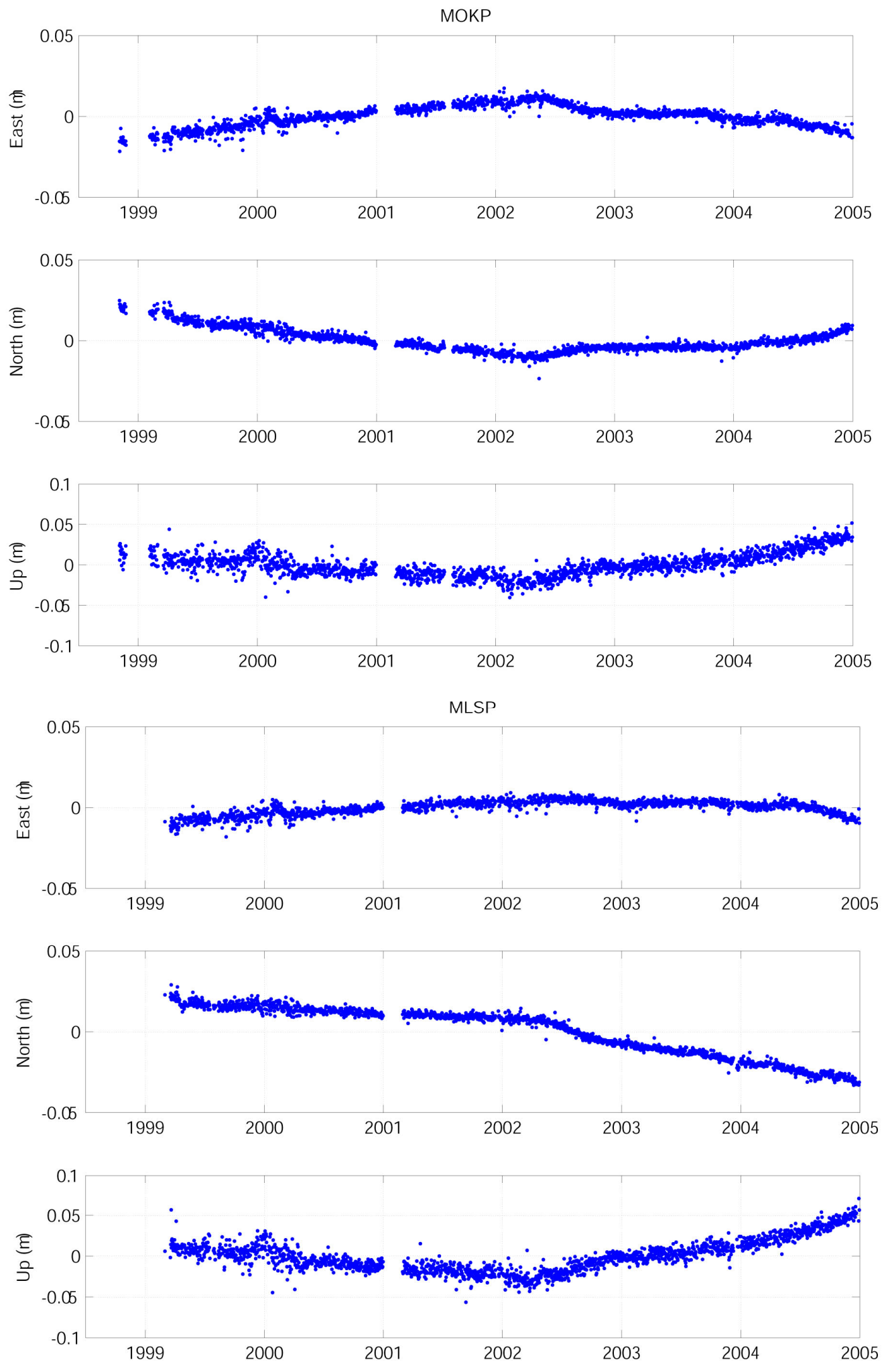

Figure 5o. Time series of continuously recording sites MLSP, MOKP. 

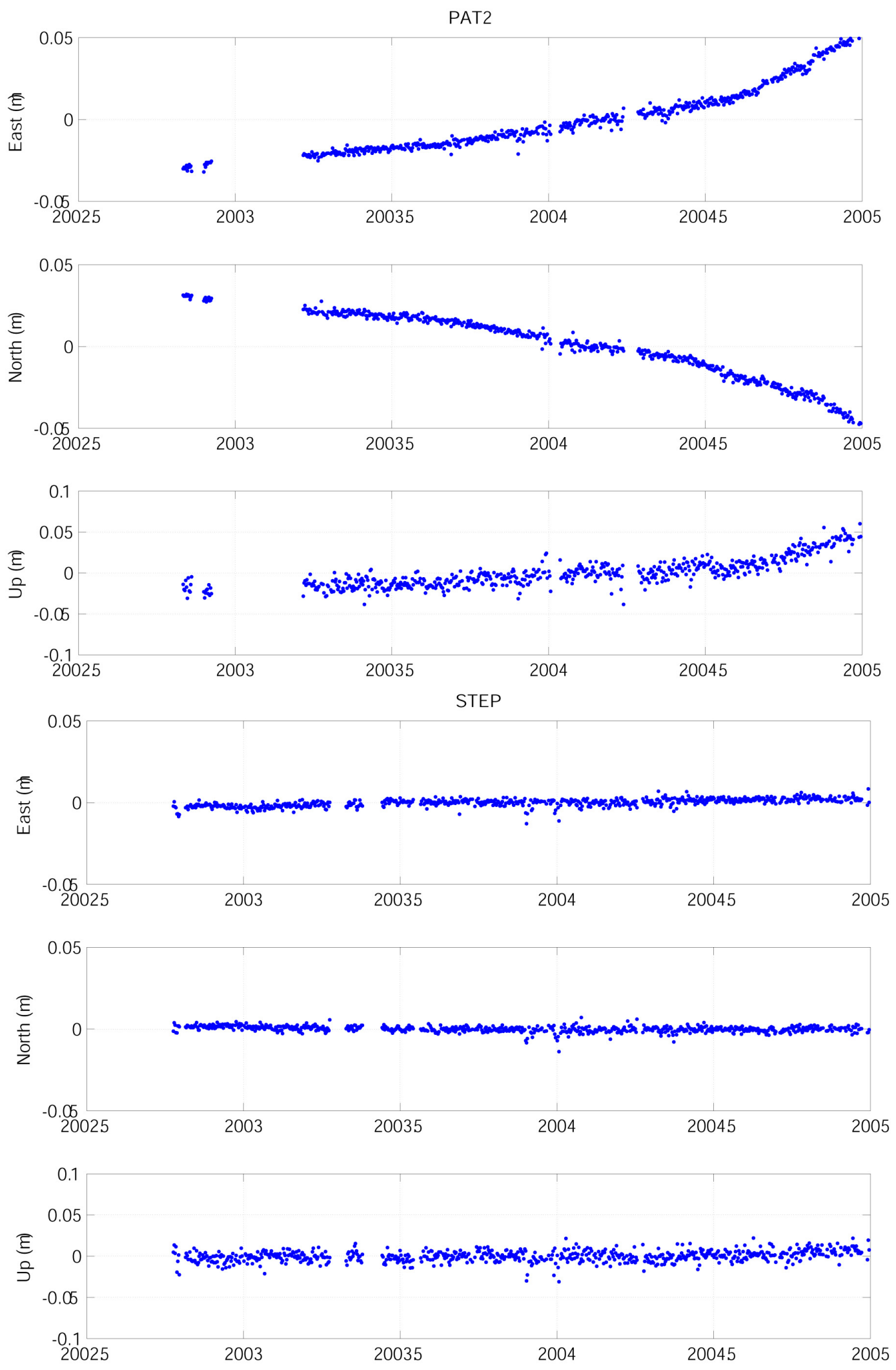

Figure $5 \mathrm{p}$. Time series of continuously recording sites PAT2, STEP. 

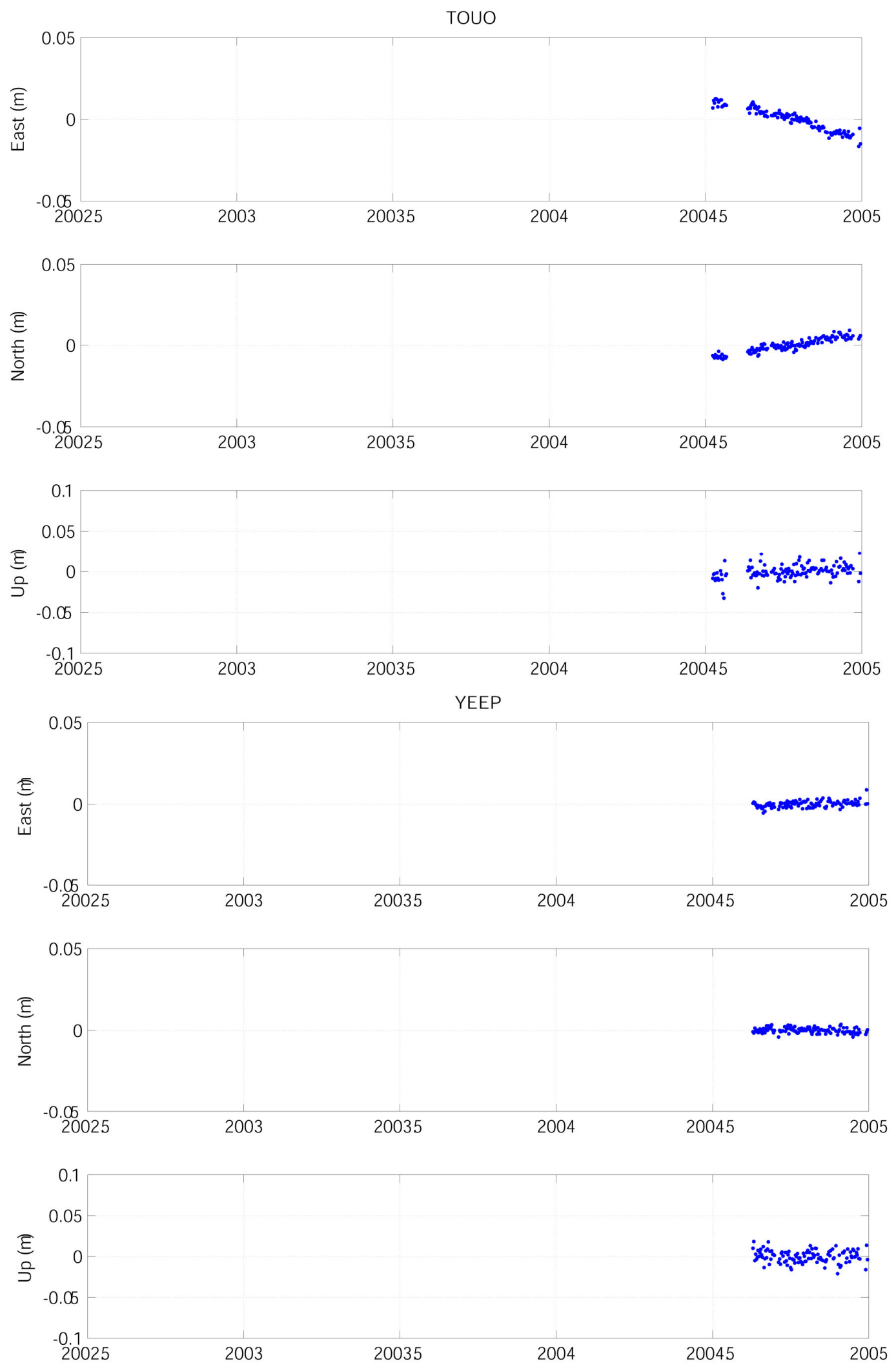

Figure 5q. Time series of continuously recording sites TOUO, YEEP. 


\section{References Cited}

Bar-Sever, Y., P. Kroger, J., Borjesson A., 1998, Estimating horizontal gradients of tropospheric path delay with a single GPS receiver, J. Geophys. Research, v.103, 5019-5035.

Cervelli, P., Miklius, A., Owen, S., Segall, P., Lisowski, M. and P. G. Okubo, 1999, Implications of the 1999 M5.9 Pahala, Hawai 'i earthquake on the tectonic structure of Kilauea and Mauna Loa volcanoes [abstr.] Eos, Transactions, American Geophysical Union, in AGU 1999 fall meeting. v. 80 , no. 46, p. 958.

Cervelli, P., Segall, P., Johnson, K., Lisowski, M., and Miklius, A., 2002a, Sudden aseismic fault slip on the south flank of Kilauea Volcano: Nature, v. 415, Feb. 28, p. 1014-1018.

Cervelli, P., Amelung, F., Owen, S., Meertens, C., Segall, P., Miklius, A., and M. Lisowski, 2002b, The September 12, 1999 Upper East Rift Zone dike intrusion at Kilauea Volcano, Hawaii, Journal of Geophysical Research, v. 107, no. B7, p. 3-13.

Cervelli, P. and Miklius, A., 2003, The Shallow Magmatic System of Kîlauea Volcano, in The Pu'u 'O'o-Kupaianaha Eruption of Kilauea Volcano, Hawai i: The First Twenty Years, Heliker, C., Swanson, D.A., and Takahashi, T.J., eds., U.S. Geological Survey Professional Paper 1676, pp. 149-164.

Delaney, P.T., Denlinger, R.P., Lisowski, M., Miklius, A., Okubo, P.G., Okamura, A.T., and Sako, M.K., 1998, Volcanic spreading at Kilauea, 1976-1996: Journal of Geophysical Research, v. 103, no. B8, p. 18,003-18,023.

Desmarais, Emily and Segall, Paul, in press, Transient deformation following the January 30, 1997dike intrusion at Kilauea Volcano, Hawaii, Journal of Geophysical Research

Dvorak, J., 1994, An Earthquake Cycle along the South Flank of Kilauea Volcano, Hawaii: Journal of Geophysical Research, v.99, p. 9533-9541.

Heliker, C., and Mattox, T. N., 2003, The first two decades of the Pu`u 'O'o-Kupaianaha eruption: chronology and selected bibliography, 2003, in Heliker, C., Swanson, D.A., and Takahashi, T. J., eds., The Pu'u 'O`o -Kupaianaha eruption of Kilauea Volcano, Hawai 'i: The first twenty years: USGS Professional Paper 1676, p. 1-28.

Larson, K.M., Cervelli, P., Lisowski, M., Miklius, A., Segall, P., and Owen, S., 2001, Volcano monitoring using the global positioning system: filtering strategies: Journal of Geophysical Research, v. 106, no. B9, p. 19,453-19,464.

Lichten, S. and Border, J., 1987, Strategies for high-precision Global Positioning System orbit determination, J. Geophys. Res., v. 92, 12,751-12,762.

Miklius, A., Iwatsubo, E.Y., Denlinger, R., Okamura, A.T., Sako, M.K., Yamashita, K., 1994, GPS measurements on the island of Hawaii in 1992, U.S. Geological Survey Open-File Report 94288, $44 \mathrm{pp}$.

Miklius, A., Coloma, F., Denlinger, R., Lisowski, M., Owen, S., Sako, M., and Segall, P., 1997, Global Positioning System measurements on the island of Hawaii: 1993 through 1996: U.S. Geological Survey Open-File Report 97-698, 114 p.

Miklius, A., Lisowski, M., Delaney, P.T., Denlinger, R.P., Dvorak, J., Okamura, A.T., Sako, M.K., 1995, Recent Inflation and Flank Movement of Mauna Loa Volcano: in Mauna Loa Revealed, American Geophysical Union Geophysical Monograph 92, p. 199-205.

Miklius, A. and Cervelli, P., 2003, Interaction between Kilauea and Mauna Loa, Nature, v. 421, p. 229.

Owen, S., Miklius, A., Segall, P., Lisowski, M., Sako, M., 1997, Displacements From the June 30, 1997, M5.5 Kilauea South Flank Earthquake and Preceding Decrease in Slip Rate [abstr.], Eos, Transactions, American Geophysical Union, in AGU 1997 fall meeting. 
Owen, S., Segall, P., Lisowski, M., Miklius, A., Murray, M., Bevis, M., and Foster, J., 2000a, January 30, 1997 eruptive event on Kilauea Volcano, Hawaii, as monitored by continuous GPS: Geophysical Research Letters, v. 27, no. 17, p. 2757-2760.

Owen, S., Segall, P., Lisowski, M., Miklius, A., Denlinger, R., and Sako, M., 2000b, Rapid deformation of Kilauea Volcano: global positioning system measurements between 1990 and 1996: Journal of Geophysical Research, v. 105, no. B8, p. 18,983-18,998.

Segall, P., Cervelli, P., Owen, S., Lisowski, M., and Miklius, A., 2001, Constraints on dike propagation from continuous GPS measurements: Journal of Geophysical Research, v. 106, no. B9, p. 19,301-19,317.

Zumberge, J. F., M. B. Heflin, D. C. Jefferson, M. M. Watkins, and F. H. Webb, 1997, Precise point positioning for the efficient and robust analysis of GPS data from large networks, $\mathrm{J}$. Geophys. Res., v. 102, p. 5005-5017. 